\title{
Parabolic Anderson Model in a Dynamic Random Environment: Random Conductances
}

\author{
D. Erhard ${ }^{1} \cdot$ F. den Hollander ${ }^{2}$ G. Maillard ${ }^{3}$
}

Received: 24 January 2016 / Accepted: 11 April 2016 / Published online: 25 April 2016

(C) The Author(s) 2016. This article is published with open access at Springerlink.com

\begin{abstract}
The parabolic Anderson model is defined as the partial differential equation $\partial u(x, t) / \partial t=\kappa \Delta u(x, t)+\xi(x, t) u(x, t), x \in \mathbb{Z}^{d}, t \geq 0$, where $\kappa \in[0, \infty)$ is the diffusion constant, $\Delta$ is the discrete Laplacian, and $\xi$ is a dynamic random environment that drives the equation. The initial condition $u(x, 0)=u_{0}(x), x \in \mathbb{Z}^{d}$, is typically taken to be non-negative and bounded. The solution of the parabolic Anderson equation describes the evolution of a field of particles performing independent simple random walks with binary branching: particles jump at rate $2 d \kappa$, split into two at rate $\xi \vee 0$, and die at rate $(-\xi) \vee 0$. In earlier work we looked at the Lyapunov exponents
\end{abstract}

$$
\lambda_{p}(\kappa)=\lim _{t \rightarrow \infty} \frac{1}{t} \log \mathbb{E}\left([u(0, t)]^{p}\right)^{1 / p}, \quad p \in \mathbb{N}, \quad \lambda_{0}(\kappa)=\lim _{t \rightarrow \infty} \frac{1}{t} \log u(0, t) .
$$

For the former we derived quantitative results on the $\kappa$-dependence for four choices of $\xi$ : space-time white noise, independent simple random walks, the exclusion process and the voter model. For the latter we obtained qualitative results under certain space-time mixing conditions on $\xi$. In the present paper we investigate what happens when $\kappa \Delta$ is replaced by $\Delta^{\mathcal{K}}$, where $\mathcal{K}=\left\{\mathcal{K}(x, y): x, y \in \mathbb{Z}^{d}, x \sim y\right\}$ is a collection of random conductances between neighbouring sites replacing the constant

D. Erhard

D.Erhard@warwick.ac.uk

1 Mathematics Department, University of Warwick, Coventry, CV4 7AL, UK

2 Mathematical Institute, Leiden University, P.O. Box 9512, 2300 RA Leiden, The Netherlands

3 CNRS, Centrale Marseille, Aix-Marseille Université, I2M, UMR 7373, 13453 Marseille, France 
conductances $\kappa$ in the homogeneous model. We show that the associated annealed Lyapunov exponents $\lambda_{p}(\mathcal{K}), p \in \mathbb{N}$, are given by the formula

$$
\lambda_{p}(\mathcal{K})=\sup \left\{\lambda_{p}(\kappa): \kappa \in \operatorname{Supp}(\mathcal{K})\right\}
$$

where, for a fixed realisation of $\mathcal{K}, \operatorname{Supp}(\mathcal{K})$ is the set of values taken by the $\mathcal{K}$ field. We also show that for the associated quenched Lyapunov exponent $\lambda_{0}(\mathcal{K})$ this formula only provides a lower bound, and we conjecture that an upper bound holds when $\operatorname{Supp}(\mathcal{K})$ is replaced by its convex hull. Our proof is valid for three classes of reversible $\xi$, and for all $\mathcal{K}$ satisfying a certain clustering property, namely, there are arbitrarily large balls where $\mathcal{K}$ is almost constant and close to any value in $\operatorname{Supp}(\mathcal{K})$. What our result says is that the annealed Lyapunov exponents are controlled by those pockets of $\mathcal{K}$ where the conductances are close to the value that maximises the growth in the homogeneous setting. In contrast our conjecture says that the quenched Lyapunov exponent is controlled by a mixture of pockets of $\mathcal{K}$ where the conductances are nearly constant. Our proof is based on variational representations and confinement arguments.

Keywords Parabolic Anderson equation · Random conductances · Lyapunov exponents · Large deviations · Variational representations · Confinement

Mathematics Subject Classification (2010) Primary 60K35, 60H25, 82C44 . Secondary 35B40, 60F10

\section{Introduction and Main Results}

Random walks with random conductances have been studied intensively in the literature. For a recent overview, we refer the reader to Biskup [2]. The goal of the present paper is to study the version of the Parabolic Anderson model where the underlying random walk is driven by random conductances, and to investigate the effect on the Lyapunov exponents.

\subsection{Parabolic Anderson Model with Random Conductances}

The parabolic Anderson model with random conductances is the partial differential equation

$$
\left\{\begin{array}{l}
\frac{\partial}{\partial t} u(x, t)=\left(\Delta^{\mathcal{K}} u\right)(x, t)+\xi(x, t) u(x, t), \quad x \in \mathbb{Z}^{d}, t \geqslant 0 . \\
u(x, 0)=u_{0}(x),
\end{array}\right.
$$


Here, $u$ is an $\mathbb{R}$-valued random field, $\Delta^{\mathcal{K}}$ is the discrete Laplacian with random conductances $\mathcal{K}$ acting on $u$ as

$$
\Delta^{\mathcal{K}} u(x, t)=\sum_{\substack{y \in \mathbb{Z}^{d} \\ y \sim x}} \mathcal{K}(x, y)[u(y, t)-u(x, t)],
$$

where $\left\{\mathcal{K}(x, y): x, y \in \mathbb{Z}^{d}, x \sim y\right\}$ is a $(0, \infty)$-valued field of random conductances, $x \sim y$ means that $x$ and $y$ are neighbours, while

$$
\xi=\left(\xi_{t}\right)_{t \geqslant 0} \text { with } \xi_{t}=\left\{\xi(x, t): x \in \mathbb{Z}^{d}\right\}
$$

is an $\mathbb{R}$-valued random field playing the role of a dynamic random environment that drives the equation. Throughout the paper we assume that

$$
0 \leqslant u_{0}(x) \leqslant 1 \quad \forall x \in \mathbb{Z}^{d} .
$$

The $\xi$-field and the $\mathcal{K}$-field are defined on probability spaces $(\Omega, \mathcal{F}, \mathbb{P})$ and $(\tilde{\Omega}, \tilde{\mathcal{F}}, \tilde{\mathbb{P}})$, respectively. Throughout the paper we assume that

$$
\begin{aligned}
& \text { (1) } 0<c \leqslant \mathcal{K}(x, y) \leqslant C<\infty \forall x, y \in \mathbb{Z}^{d}, x \sim y \text {. } \\
& \text { (2) } \mathcal{K}(x, y)=\mathcal{K}(y, x) \forall x, y \in \mathbb{Z}^{d}, x \sim y .
\end{aligned}
$$

The formal solution of (1.1) is given by the Feynman-Kac formula

$$
u(x, t)=E_{x}\left(\exp \left\{\int_{0}^{t} \xi\left(X^{\mathcal{K}}(s), t-s\right) d s\right\} u_{0}\left(X^{\mathcal{K}}(t)\right)\right),
$$

where $X^{\mathcal{K}}=\left(X^{\mathcal{K}}(t)\right)_{t>0}$ is the continuous-time Markov process with generator $\Delta^{\mathcal{K}}$, and $P_{X}$ is the law of $X^{\mathcal{K}}$ given $X^{\mathcal{K}}(0)=x$. When $\mathcal{K} \equiv \kappa \in(0, \infty)$, we write $X^{\mathcal{K}}=X^{\kappa}$. In Section 1.3 we will show that under mild assumptions on $\xi$ the formula in (1.6) is the unique non-negative solution of (1.1). These assumptions are fulfilled for the three classes of $\xi$ that will receive special attention in our paper, which we list next.

\subsection{Choices of Dynamic Random Environments}

(I) Space-time White Noise Here $\xi$ is the Markov process on $\Omega=\mathbb{R}^{\mathbb{Z}^{d}}$ given by

$$
\xi(x, t)=\frac{\partial}{\partial t} W(x, t),
$$

where $W=\left(W_{t}\right)_{t \geq 0}$ with $W_{t}=\left\{W(x, t): x \in \mathbb{Z}^{d}\right\}$ is a field of independent standard Brownian motions, and (1.1) is to be understood as an Itô-equation.

\section{(II) Independent Random Walks}

(IIa) Finite System Here $\xi$ is the Markov process on $\Omega=\{0, \ldots, n\}^{\mathbb{Z}^{d}}$ given by

$$
\xi(x, t)=\sum_{k=1}^{n} \delta_{x}\left(Y_{k}^{\rho}(t)\right)
$$


where $\left\{Y_{k}^{\rho}: 1 \leq k \leq n\right\}$ is a collection of $n \in \mathbb{N}$ independent continuous-time simple random walks jumping at rate $2 d \rho$ and starting at the origin.

(IIb) Infinite System Here $\xi$ is the Markov process on $\Omega=\mathbb{N}_{0}^{\mathbb{Z}^{d}}$ given by

$$
\xi(x, t)=\sum_{y \in \mathbb{Z}^{d}} \sum_{j=1}^{N_{y}} \delta_{x}\left(Y_{j}^{y}(t)\right),
$$

where $\left\{Y_{j}^{y}: y \in \mathbb{Z}^{d}, 1 \leq j \leq N_{y}, Y_{j}^{y}(0)=y\right\}$ is an infinite collection of independent continuous-time simple random walks jumping at rate $2 d$, and $\left(N_{y}\right)_{y \in \mathbb{Z}^{d}}$ is a Poisson random field with intensity $v \in(0, \infty)$. The generator $L$ of this process is defined as follows (see Andjel [1]). Let $l(x)=e^{-\|x\|}, x \in \mathbb{Z}^{d}$, with $\|\cdot\|$ the Euclidean norm. Define the $l$-norm on $\Omega$ as

$$
\|\eta\|_{l}=\sum_{x \in \mathbb{Z}^{d}} \eta(x) l(x),
$$

and define the sets $\mathcal{E}_{l}=\left\{\eta \in \Omega:\|\eta\|_{l}<\infty\right\}$ and $\mathcal{L}_{l}=\left\{f: \mathcal{E}_{l} \rightarrow\right.$ $\mathbb{R}$ Lipschitz continuous\}. Then $L$ acts on $f \in \mathcal{L}_{l}$ as

$$
(L f)(\eta)=\sum_{x \in \mathbb{Z}^{d}} \sum_{\substack{y \in \mathbb{Z}^{d} \\ y \sim x}} \eta(x)\left[f\left(\eta^{x, y}\right)-f(\eta)\right],
$$

and $\eta^{x, y}$ is defined by

$$
\eta^{x, y}(z)=\left\{\begin{array}{l}
\eta(z), \quad z \neq x, y \\
\eta(x)-1, \quad z=x \\
\eta(y)+1, \quad z=y
\end{array}\right.
$$

Write $\mu$ for the Poisson random field with intensity $v$. This is the invariant distribution of the dynamics.

(III) Spin-flip Systems Here $\xi$ is the Markov process on $\Omega=\{0,1\}^{\mathbb{Z}^{d}}$ whose generator $L$ acts on cylinder functions $f$ as (see Liggett [15, Chapter III])

$$
(L f)(\eta)=\sum_{x \in \mathbb{Z}^{d}} c(x, \eta)\left[f\left(\eta^{x}\right)-f(\eta)\right],
$$

where, for a configuration $\eta, c(x, \eta)$ is the rate for the spin at $x$ to flip, and

$$
\eta^{x}(z)= \begin{cases}\eta(z), & z \neq x \\ 1-\eta(x), & z=x .\end{cases}
$$

We assume that the rates $c(x, \eta)$ are such that

(i) $\xi$ is ergodic and reversible, i.e., there is a probability distribution $\mu$ on $\Omega$ such that $\xi_{t}$ converges to $\mu$ in distribution as $t \rightarrow \infty$ for any choice of $\xi_{0} \in \Omega$, and $c(x, \eta) \mu(d \eta)=c\left(x, \eta^{x}\right) \mu\left(d \eta^{x}\right)$ for all $\eta \in \Omega$ and $x \in \mathbb{Z}^{d}$.

(ii) $\xi$ is attractive, i.e., $c(x, \eta) \leq c(x, \zeta)$ for all $\eta \leq \zeta$ when $\eta(x)=\zeta(x)=0$ and $c(x, \eta) \geq c(x, \zeta)$ for all $\eta \leq \zeta$ when $\eta(x)=\zeta(x)=1$ (where we write $\eta \leq \zeta$ when $\eta(x) \leq \zeta(x)$ for all $\left.x \in \mathbb{Z}^{d}\right)$. 
We further assume that

(iii) $\xi_{0}$ has distribution $\mu$.

Let $\mathcal{M}$ be the class of continuous non-decreasing functions $f$ on $\Omega$, the latter meaning that $f(\eta) \leq f(\zeta)$ for all $\eta \leq \zeta$. As shown in Liggett [15, Theorems II.2.14 and III.2.13], attractive spin-flip systems preserve the FKG-inequality, i.e., if $\xi_{0}$ satisfies the FKG-inequality (e.g. if $\xi_{0}$ is distributed according to $\mu$ ), then so does $\xi_{t}$ for all $t \geq 0$, i.e.,

$$
\mathbb{E}\left(f\left(\xi_{t}\right) g\left(\xi_{t}\right)\right) \geqslant \mathbb{E}\left(f\left(\xi_{t}\right)\right) \mathbb{E}\left(g\left(\xi_{t}\right)\right) \quad \forall f, g \in \mathcal{M} .
$$

Examples include the ferromagnetic stochastic Ising model, for which

$$
c(x, \eta)=\exp \left[-\beta \sum_{\substack{y \in \mathbb{Z}^{d} \\ y \sim x}} \sigma(x) \sigma(y)\right], \quad \sigma(x)=2 \eta(x)-1 \in\{-1,+1\},
$$

with $\beta \in(0, \infty)$ the inverse temperature. This dynamics has at least one invariant distribution. It is shown in Liggett [15, Theorem IV.2.3 and Proposition IV.2.7] that any reversible spin-flip system is a stochastic Ising model for some interaction potential (not necessarily between neighbours).

\subsection{Lyapunov Exponents}

Our focus will be on the annealed Lyapunov exponents

$$
\lambda_{p}(\mathcal{K})=\lim _{t \rightarrow \infty} \frac{1}{t} \log \mathbb{E}\left([u(0, t)]^{p}\right)^{1 / p}, \quad p \in \mathbb{N},
$$

and the quenched Lyapunov exponent

$$
\lambda_{0}(\mathcal{K})=\lim _{t \rightarrow \infty} \frac{1}{t} \log u(0, t),
$$

provided the limits exist. Note that

$-\mathcal{K}$ is fixed, i.e., the annealing and the quenching is with respect to $\xi$ only.

We write $\lambda_{p}(\kappa)$ when $\mathcal{K} \equiv \kappa$.

Let $\mathcal{E}^{d}$ be the edge set of $\mathbb{Z}^{d}$, and let $\operatorname{Supp}(\mathcal{K})=\left\{\mathcal{K}(x, y):(x, y) \in \mathcal{E}^{d}\right\}$, i.e., $\operatorname{Supp}(\mathcal{K})$ is the union over all edges $(x, y) \in \mathcal{E}^{d}$ of the values taken by the marginals $\mathcal{K}(x, y)$. For $x \in \mathbb{Z}^{d}$ and $t>0$, let

$$
B_{t}(x)=x+\left([-t, t]^{d} \cap \mathcal{E}^{d}\right)
$$

be the edges in the box of radius $t$ centered at $x$.

Definition 1.1 We say that $\mathcal{K}$ has the clustering property when for all $\kappa \in \operatorname{Supp}(\mathcal{K})$, $\delta>0$ and $t>0$ there exist radii $L_{\delta, \kappa}(t)$, satisfying $\lim _{t \rightarrow \infty} L_{\delta, \kappa}(t)=\infty$, and centers $x(\kappa, \delta, t) \in \mathbb{Z}^{d}$, satisfying $\lim _{t \rightarrow \infty}\|x(\kappa, \delta, t)\| / t=0$, such that $\mathcal{K}(y, z) \in$ $(\kappa-\delta, \kappa+\delta) \cap \operatorname{Supp}(\mathcal{K})$ for all $(y, z) \in B_{L_{\delta, \kappa}(t)}(x(\kappa, \delta, t))$. 
For the binary case $\operatorname{Supp}(\mathcal{K})=\left\{\kappa_{1}, \kappa_{2}\right\}$, the clustering property states that there are two sequences of boxes $B^{1}(t)$ and $B^{2}(t)$, whose sizes tend to infinity and whose distances to the origin are $o(t)$, such that $\mathcal{K}(x, y)=\kappa_{1}$ for all $(x, y) \in B^{1}(t)$ and $\mathcal{K}(x, y)=\kappa_{2}$ for all $(x, y) \in B^{2}(t)$. Note that if $\mathcal{K}$ is i.i.d., then it has the clustering property with probability 1 .

Our main result for the annealed Lyapunov exponents is the following.

Theorem 1.2 Let $\xi$ be as in (I)-(III), and let $\mathcal{K}$ have the clustering property. Then for all $p \in \mathbb{N}$ the limit in (1.17) exists and equals

$$
\lambda_{p}(\mathcal{K})=\sup \left\{\lambda_{p}(\kappa): \kappa \in \operatorname{Supp}(\mathcal{K})\right\}, \quad p \in \mathbb{N} .
$$

This equality holds irrespective of whether the right-hand side is finite or infinite. Moreover, $\lambda_{p}(\mathcal{K})$ is continuous, non-increasing and convex in each of the components of $\mathcal{K}$ on any open domain where it is finite.

To obtain a similar result for the quenched Lyapunov exponent, we need to make a different set of assumptions on $\xi$ :

(1) $\xi$ is stationary and ergodic under translations in space and time.

(2) $\xi$ is not constant and $\mathbb{E}(|\xi(0,0)|)<\infty$.

(3) $s \mapsto \xi(x, s)$ is locally integrable for every $x \in \mathbb{Z}^{d}$, $\xi$-a.s.

(4) $\mathbb{E}\left(e^{q \xi(0,0)}\right)<\infty$ for all $q \in \mathbb{R}$.

As a consequence of Assumptions (1)-(4), (1.1) has a unique non-negative solution given by (1.6) (see Erhard, den Hollander and Maillard [8]). The dynamics in (I)(III) satisfy (1)-(4). More examples may be found in [8, Corollary 1.19].

Theorem 1.3 Suppose that $u(x, 0)=\delta_{0}(x)$. Let $\xi$ satisfy $(1)-(4)$, and let $\mathcal{K}$ have the clustering property. Then the limit in (1.18) exists $\mathbb{P}$-a.s. and in $\mathbb{P}$-mean and satisfies

$$
\lambda_{0}(\mathcal{K}) \geqslant \sup \left\{\lambda_{0}(\kappa): \kappa \in \operatorname{Supp}(\mathcal{K})\right\} .
$$

This inequality holds irrespective of whether the right-hand side is finite or infinite.

\subsection{Discussion and Outline}

1. Theorem 1.2 shows that, in the annealed setting, the clustering strategy wins over the non-clustering strategy, i.e., the annealed Lyapunov exponents are controlled by those pockets in $\mathcal{K}$ where the conductances are close to the value that maximises the growth in the homogeneous setting, i.e., mixed pockets in $\mathcal{K}$ are subdominant. For the quenched Lyapunov exponent this is not expected to be the case, see also Item 4 below. For the annealed Lyapunov exponents we can use variational representations, for the quenched Lyapunov exponent the argument is more delicate.

2. Examples (I) and (III) are non-conservative dynamics. Examples (IIa)-(IIb) are conservative dynamics. All are reversible. 
3. For $\mathcal{K} \equiv \kappa$, the annealed Lyapunov exponents $\lambda_{p}(\kappa), p \in \mathbb{N}$, are known to be continuous, non-increasing and convex in $\kappa$ when finite, for each of the choices in (I)-(III). Hence (1.20) reduces to

$$
\lambda_{p}(\mathcal{K})=\lambda_{p}\left(\kappa_{*}\right), \quad \kappa_{*}=\operatorname{essinf}[\operatorname{Supp}(\mathcal{K})], \quad p \in \mathbb{N},
$$

i.e., the annealed growth is dominated by the pockets with the slowest conductances.

4. The quenched Lyapunov exponent $\lambda_{0}(\kappa)$ is continuous in $\kappa$ as well, but it fails to be non-increasing (it is expected to be unimodal). Hence we do not expect the inequality in (1.21) to be an equality, as in the annealed case. In Section 5 we provide an illustrative example for a decorated version of $\mathbb{Z}^{d}$, i.e., each pair of neighbouring sites of $\mathbb{Z}^{d}$ is connected by two edges rather than one, for which the inequality in (1.21) is strict. We conjecture that the following upper bound holds.

Conjecture 1.4 Under the conditions of Theorem 1.3,

$$
\lambda_{0}(\mathcal{K}) \leq \sup \left\{\lambda_{0}(\kappa): \kappa \in \operatorname{Conv}(\operatorname{Supp}(\mathcal{K}))\right\},
$$

where $\operatorname{Conv}(\operatorname{Supp}(\mathcal{K}))$ is the convex hull of $\operatorname{Supp}(\mathcal{K})$.

5. The Feynman-Kac formula shows that understanding the Lyapunov exponents amounts to understanding the large deviation behaviour of the integral of the $\xi$-field along the trajectory of a random walk in random environment. Drewitz [6] studies the case where $\Delta$ is replaced by a Laplacian with a deterministic drift and $\xi$ is constant in time. It is proven that the Lyapunov exponent is maximal when the drift is zero.

6. We expect that pushing the method of our proof a bit further one may relax the boundedness and uniform ellipticity assumption (1.5) on the $\mathcal{K}$-field. However, at this point this seems only a technical issue and does not provide much more insight so that we refrained from doing so.

Outline The outline of the remainder of the paper is as follows. In Section 2 we derive variational formulas for the annealed Lyapunov exponents and use these to derive the rightmost inequality in (2.2), i.e., $\leq$ in (1.20) and the monotonicity in each coordinate of $\mathcal{K}$. In Section 3 we derive the leftmost inequality in (2.2), i.e., $\geq$ in (1.20). The proof uses a confinement approximation, showing that the annealed Lyapunov exponent does not change when the random walk in the Feynman-Kac formula (1.6) is confined to a slowly growing box. In Section 4 we turn to the quenched Lyapunov exponent and prove the lower bound in Theorem 1.3 with the help of a confinement approximation. In Section 5 we discuss the failure of the corresponding upper bound by providing a counterexample for a decorated lattice.

In Appendix A we show that the annealed Lyapunov exponents are the same for all initial conditions that are bounded. In Appendix A we prove a technical lemma about the generator of dynamics (IIb). 


\section{Annealed Lyapunov Exponents: Preparatory Facts, Variational Representations, Existence and Upper Bound}

Section 2.1 contains some preparatory facts. Section 2.2 gives variational representations for $\lambda_{p}(\mathcal{K})$ for each of the four dynamics (Propositions 2.2-2.5 below) and settles the existence. Section 2.3 explains why these variational representations imply the upper bound. Section 2.4 provides the proof of the variational representations.

\subsection{Preparatory Facts}

The following proposition, whose proof is deferred to Appendix A, shows that the annealed Lyapunov exponents are the same for any bounded initial condition $u_{0}$, i.e., without loss of generality we may take $u_{0}=\delta_{0}$ or $u_{0} \equiv 1$.

Proposition 2.1 Fix $p \in \mathbb{N}$ and $\kappa>0$. Let $\xi$ be as in (I)-(III), and let $\lambda_{p}^{\delta_{0}}(\kappa)$ and $\lambda_{p}^{\mathbb{1}}(\kappa)$ be the $p$-th annealed Lyapunov exponent for $u_{0}=\delta_{0}$ and $u_{0} \equiv 1$, respectively. Then

$$
\lambda_{p}^{\delta_{0}}(\kappa)=\lambda_{p}^{\mathbb{1}}(\kappa)
$$

Consequently, the proof of Theorem 1.2 reduces to the following two inequalities:

$$
\sup \left\{\lambda_{p}^{\delta_{0}}(\kappa): \kappa \in \operatorname{Supp}(\mathcal{K})\right\} \leqslant \lambda_{p}^{\delta_{0}}(\mathcal{K}), \quad \lambda_{p}^{\mathbb{1}}(\mathcal{K}) \leqslant \sup \left\{\lambda_{p}^{\mathbb{1}}(\kappa): \kappa \in \operatorname{Supp}(\mathcal{K})\right\} .
$$

We prove the second inequality (upper bound) in the present section and the first inequality (lower bound) in Section 3. For ease of notation we suppress the upper index from the respective Lyapunov exponents.

Before we proceed we make three observations:

(I) For $\xi$ space-time white noise, it follows from Carmona and Molchanov [3, Theorem II.3.2] that

$$
\mathbb{E}\left([u(0, t)]^{p}\right)=E_{0}^{\otimes p}\left(\exp \left\{\sum_{1 \leqslant i<j \leqslant p} \int_{0}^{t} 1\left\{\left\{X_{i}^{\mathcal{K}}(s)=X_{j}^{\mathcal{K}}(s)\right\} d s\right\} \prod_{i=1}^{p} u_{0}\left(X_{i}^{\mathcal{K}}(t)\right)\right),\right.
$$

where $E_{0}^{\otimes p}$ is the expectation with respect to $p$ independent simple random walks $X_{1}^{\mathcal{K}}, \ldots, X_{p}^{\mathcal{K}}$, all having generator $\Delta^{\mathcal{K}}$ and all starting at 0 .

(IIa) For $\xi$ finite independent simple random walks we have

$$
\mathbb{E}\left([u(0, t)]^{p}\right)=\left(E_{0}^{\otimes p} \otimes E_{0}^{\otimes n}\right)\left(\exp \left\{\sum_{i=1}^{p} \sum_{j=1}^{n} \int_{0}^{t} \mathbb{1}\left\{X_{i}^{\mathcal{K}}(s)=X_{j}^{\rho}(s)\right\} d s\right\} \prod_{i=1}^{p} u_{0}\left(X_{i}^{\mathcal{K}}(t)\right)\right),
$$

which is similar to (2.3). In particular, the proof of the upper bound in Theorem 1.2 is similar for (I) and (IIa). Therefore we will only give the proof for (IIa). 
(I)-(III) are reversible, and so we have

$$
u(0, t)=E_{0}\left(\exp \left\{\int_{0}^{t} \xi\left(X^{\mathcal{K}}(s), s\right) d s\right\} u_{0}\left(X^{\mathcal{K}}(t)\right)\right)
$$

in $\mathbb{P}$-distribution.

\subsection{Variational Representations}

We assume that $u_{0} \equiv 1$. For $p \in \mathbb{N}, i \in\{1, \ldots, p\}, x=\left(x_{1}, \ldots, x_{p}\right) \in \mathbb{Z}^{d p}$ and $y \in \mathbb{Z}^{d}$, write $\left.f(x)\right|_{x_{i} \rightarrow y}$ to denote $f(x)$ but with the argument $x_{i}$ replaced by $y$.

Proposition 2.2 Let $\xi$ be as in (I). Then, for all $p \in \mathbb{N}$,

$$
\lambda_{p}(\mathcal{K})=\frac{1}{p} \sup _{\|f\|_{l^{2}\left(\mathbb{Z}^{d p}\right)}=1}\left\{A_{1}(f)-A_{2}(f)\right\}
$$

where

$$
\begin{aligned}
& A_{1}(f)=\sum_{x \in \mathbb{Z}^{d p}} \sum_{1 \leqslant i<j \leqslant p} \delta_{0}\left(x_{i}, x_{j}\right) f(x)^{2}, \\
& A_{2}(f)=\frac{1}{2} \sum_{x \in \mathbb{Z}^{d p}} \sum_{i=1}^{p} \sum_{\substack{z \in \mathbb{Z}^{d} \\
z \sim x_{i}}} \mathcal{K}\left(x_{i}, z\right)\left[\left.f(x)\right|_{x_{i} \rightarrow z}-f(x)\right]^{2} .
\end{aligned}
$$

Proposition 2.3 Let $\xi$ be as in (IIa). Then, for all $p \in \mathbb{N}$,

$$
\lambda_{p}(\mathcal{K})=\frac{1}{p} \sup _{\|f\|_{l^{2}\left(\mathbb{Z}^{d p} \times \mathbb{Z}^{d n}\right)}=1}\left\{A_{1}(f)-A_{2}(f)-A_{3}(f)\right\},
$$

where

$$
\begin{aligned}
& A_{1}(f)=\sum_{x \in \mathbb{Z}^{d p}} \sum_{y \in \mathbb{Z}^{d n}} \sum_{i=1}^{p} \sum_{j=1}^{n} \delta_{0}\left(x_{i}, y_{j}\right) f(x, y)^{2}, \\
& A_{2}(f)=\frac{1}{2} \sum_{x \in \mathbb{Z}^{d p}} \sum_{y \in \mathbb{Z}^{d n}} \sum_{i=1}^{p} \sum_{\substack{z \in \mathbb{Z}^{d} \\
z \sim x_{i}}} \mathcal{K}\left(x_{i}, z\right)\left[\left.f(x, y)\right|_{x_{i} \rightarrow z}-f(x, y)\right]^{2}, \\
& A_{3}(f)=\frac{\rho}{2} \sum_{x \in \mathbb{Z}^{d p}} \sum_{y \in \mathbb{Z}^{d n}} \sum_{j=1}^{n} \sum_{\substack{z \in \mathbb{Z}^{d} \\
z \sim y_{j}}}^{n}\left[\left.f(x, y)\right|_{y_{j} \rightarrow z}-f(x, y)\right]^{2} .
\end{aligned}
$$


Proposition 2.4 Fix $p \in \mathbb{N}$. Let $\xi$ be as in (IIb) and let $G(0)$ be the Green function at the origin of simple random walk jumping at rate $2 d$. Then, for all $0<p<1 / G(0)$,

$$
\lambda_{p}(\mathcal{K})=\frac{1}{p} \sup _{N \in \mathbb{N}} \sup _{\|f\|_{L^{2}(\mu \otimes m)=1}}\left\langle\left(L+\sum_{i=1}^{p} \Delta_{i}^{\mathcal{K}}+V_{N}\right) f, f\right\rangle,
$$

where

$$
\left(\Delta_{i}^{\mathcal{K}} f\right)(\eta, y)=\sum_{\substack{z \in \mathbb{Z}^{d} \\ z \sim y_{i}}} \mathcal{K}\left(y_{i}, z\right)\left[\left.f(\eta, y)\right|_{y_{i} \rightarrow z}-f(\eta, y)\right],
$$

$\mu=\otimes_{i \in \mathbb{Z}^{d}} \mathrm{POI}(v)$ is the Poisson random field with intensity $v \in(0, \infty), m$ is the counting measure on $\mathbb{Z}^{d}$, and $V_{N}: \mathbb{N}_{0}^{\mathbb{Z}^{d}} \times \mathbb{Z}^{p d} \rightarrow \mathbb{R}$ is the truncated function given by

$$
V_{N}(\eta, x)=\sum_{i=1}^{p}\left[N \wedge \eta\left(x_{i}\right)\right]
$$

and L acts on $f$ solely on its first coordinate.

Proposition 2.5 Let $\xi$ be as in (III). Then, for all $p \in \mathbb{N}$,

$$
\lambda_{p}(\mathcal{K})=\frac{1}{p} \sup _{\left.\|f\|_{L^{2}(\mu \otimes m}\right)=1}\left\{A_{1}(f)-A_{2}(f)-A_{3}(f)\right\},
$$

where $m^{p}$ is the counting measure on $\mathbb{Z}^{d p}$, and

$$
\begin{aligned}
& A_{1}(f)=\int_{\Omega} \mu(d \eta) \sum_{x \in \mathbb{Z}^{d p}} \sum_{i=1}^{p} \eta\left(x_{i}\right) f(\eta, x)^{2}, \\
& A_{2}(f)=\frac{1}{2} \int_{\Omega} \mu(d \eta) \sum_{x \in \mathbb{Z}^{d p}} \sum_{i=1}^{p} \sum_{\substack{y \in \mathbb{Z}^{d} \\
y \sim x_{i}}} \mathcal{K}\left(x_{i}, y\right)\left[\left.f(\eta, x)\right|_{x_{i} \rightarrow y}-f(\eta, x)\right]^{2}, \\
& A_{3}(f)=\frac{1}{2} \int_{\Omega} \mu(d \eta) \sum_{x \in \mathbb{Z}^{d p}} \sum_{y \in \mathbb{Z}^{d}} c(y, \eta)\left[f\left(\eta^{y}, x\right)-f(\eta, x)\right]^{2} .
\end{aligned}
$$

\subsection{Proof of the Upper Bound in Theorem 1.2}

Let $\xi$ be as in (I), (IIa), (III) or as in (IIb) with $0<p<1 / G(0)$. By Propositions $2.2-2.5, \lambda_{p}(\mathcal{K})$ is a continuous, non-increasing and convex function of the components of $\mathcal{K}$. Moreover, Propositions 2.2-2.5 are still true when $\mathcal{K}=\kappa \in(0, \infty)$. It therefore follows that $\lambda_{p}(\mathcal{K}) \leq \sup \left\{\lambda_{p}(\kappa): \kappa \in \operatorname{Supp}(\mathcal{K})\right\}$. If $\xi$ is as in (IIb) but with $p \geq 1 / G(0)$, then by [9, Theorem 1.4] the annealed Lyapunov exponents $\lambda_{p}(\kappa)$ are infinite for all $p \in \mathbb{N}$ and $\kappa \in[0, \infty)$. Hence, the upper bound in Theorem 1.2 trivially holds in this case. 


\subsection{Proof of Propositions 2.2-2.5}

The proofs are, besides the proof of $\leq$ in (2.10), essentially straightforward extensions of the proofs of [3, Lemma III.1.1], [4, Proposition 2.1] and [10, Proposition 2.2.2] for $\mathcal{K} \equiv \kappa \in(0, \infty)$. We only indicate the main steps (and so the arguments in this section are not self-contained).

\subsubsection{Proof of Propositions 2.2, 2.3 and 2.5}

Proof As mentioned in Section 2.1, the Feynman-Kac formulas for the annealed Lyapunov exponents for white noise and finitely many independent random walks are similar, since the term

$$
\sum_{1 \leqslant i<j \leqslant p} \int_{0}^{t} \mathbb{1}\left\{X_{i}^{\mathcal{K}}(s)=X_{j}^{\mathcal{K}}(s)\right\} d s
$$

in (2.3) for white noise is replaced by the term

$$
\sum_{i=1}^{p} \sum_{j=1}^{n} \int_{0}^{t} 11\left\{X_{i}^{\mathcal{K}}(s)=X_{j}^{\rho}(s)\right\} d s
$$

in (2.4) for finitely many independent random walks. Therefore a slight adaptation of the proof of Proposition 2.3 below is enough to get the corresponding result for $\xi$ being space-time white noise, i.e., $\xi$ being as in (I).

The proofs of Propositions 2.3, and 2.5 follow the same line of argument as the proofs of [4, Proposition 2.1] and [10, Proposition 2.2.1], respectively, for $\mathcal{K} \equiv \kappa$. Below we detail how to adapt the proofs. Consider the Markov process $Y=(Y(t))_{t \geq 0}$ with generator

$$
G_{V}^{\mathcal{K}}=\left\{\begin{array}{lll}
L_{1}+\sum_{i=1}^{p} \Delta_{i}^{\mathcal{K}}+V_{1} & \text { on } \ell^{2}\left(m^{n} \otimes m^{p}\right), & \text { if } \xi \text { is as in (IIa), } \\
L_{2}+\sum_{i=1}^{p} \Delta_{i}^{\mathcal{K}}+V_{2} & \text { on } L^{2}\left(\mu \otimes m^{p}\right), & \text { if } \xi \text { is as in (III), }
\end{array}\right.
$$

where $L_{1}$ and $L_{2}$ are the generators of (IIa) and (III) respectively, $\Delta_{i}^{\mathcal{K}}$ is given as in (2.11) but acting on the second coordinate of $f \in \ell^{2}\left(m^{n} \otimes m^{p}\right)$ and $f \in L^{2}\left(\mu \otimes m^{p}\right)$ (if $\xi$ is as in (IIa) and (III) respectively), and $V_{1}$ (as in $[4,(16)]$ ) and $V_{2}$ (as in $[10,(2.2 .2)])$ by

$V_{1}(x, y)=\sum_{i=1}^{n} \sum_{j=1}^{p} \delta_{0}\left(x_{j}-y_{i}\right), \quad x=\left(x_{1}, \cdots, x_{p}\right) \in \mathbb{Z}^{d p}, y=\left(y_{1}, \cdots, y_{n}\right) \in \mathbb{Z}^{d n}$,

and

$$
V_{2}(\eta, x)=\sum_{i=1}^{p} \eta\left(x_{i}\right), \quad \eta \in \Omega, x=\left(x_{1}, \cdots, x_{p}\right) \in \mathbb{Z}^{d p} .
$$

Since $L_{1}$ and $L_{2}$ are self-adjoint and bounded, and $\mathcal{K}$ has compact support and is symmetric, $G_{V}^{\mathcal{K}}$ is a bounded self-adjoint operator. 


\section{Upper Bound Let}

$$
\kappa^{*}=\operatorname{ess} \sup [\operatorname{supp}(\mathcal{K})], \quad \kappa_{*}=\operatorname{ess} \inf [\operatorname{supp}(\mathcal{K})],
$$

and let $B_{R}(t) \subset \mathbb{Z}^{d}$ be the box of radius $R(t)=t \log t$ centered at the origin. Then, for any fixed realization of $\mathcal{K}$, we have

$$
P_{0}\left(X^{\mathcal{K}}(t) \notin B_{R(t)}\right) \leq P\left(N\left(2 d \kappa^{*} t\right) \geq R(t)\right) \leq \exp \left[-C\left(d, \kappa^{*}\right) R(t)\right]
$$

for some $C\left(d, \kappa^{*}\right)>0$, where $N\left(2 d \kappa^{*} t\right)$ is Poisson distributed with parameter $2 d \kappa^{*} t$. Thus, $\lim _{t \rightarrow \infty} \frac{1}{t} \log P_{0}\left(X^{\mathcal{K}}(t) \notin B_{R(t)}\right)=-\infty$. Consequently, by a cost of a superexponentially small error, it is enough to consider in the Feynman-Kac representation (1.6) only random walk paths that stay in $B_{R}(t)$ until time $t$. This allows to use the spectral theorem as in [10, Proposition 2.2.1]. We omit the details.

Lower Bound Since $\mathcal{K}$ is bounded away from zero and infinity, it follows that for any finite $K \subset \mathbb{Z}^{d}$ there exists $C>0$ such that

$$
P_{0}\left(X^{\mathcal{K}}(1)=x\right) \geq\left(\frac{\kappa_{*}}{2 d \kappa^{*}}\right)^{\|x\|} e^{-2 d \kappa^{*}} \frac{\left(2 d \kappa_{*}\right)^{\|x\|}}{\|x\| !} \geqslant C . \quad \forall x \in K .
$$

Let $\delta>0$ and take $f_{\delta}$ such that inserted in the right hand side of (2.8) (respectively of (2.13)) it approximates the corresponding supremum in (2.8) (respectively in (2.13)) up to a difference $\delta$. It was argued in [10, Proposition 2.2.1] that there is a finite set $K_{\delta}$ such that $0 \leq f_{\delta} \leq \mathbb{1}_{K_{\delta}}$. Picking $K=K_{\delta}, \delta>0$, we get, as in [10, Eq. (2.2.10)],

$$
\begin{aligned}
E_{0}^{\otimes p} \otimes E_{0}^{\otimes n} & \left(\exp \left\{\int_{0}^{t} V_{1}(Y(s)) d s\right\}\right) \\
& \geqslant\left(C_{\delta}^{\mathcal{K}}\right)^{p}\left(C_{\delta}^{\rho}\right)^{n} \sum_{\substack{x_{1}, \ldots, x_{p} \in K_{\delta} \\
y_{1}, \ldots, y_{n} \in K_{\delta}}} E_{x_{1}, \ldots, x_{p}} \otimes E_{y_{1}, \ldots, y_{n}}\left(\exp \left\{\int_{0}^{t-1} V_{1}(Y(s)) d s\right\}\right),
\end{aligned}
$$

if $\xi$ is as in (IIa), and

$$
\mathbb{E}_{\mu, 0, \ldots, 0}\left(\exp \left\{\int_{0}^{t} V_{2}(Y(s)) d s\right\}\right) \geqslant\left(C_{\delta}^{\mathcal{K}}\right)^{p} \sum_{x_{1}, \ldots, x_{p} \in K_{\delta}} \mathbb{E}_{\mu, x_{1}, \ldots, x_{p}}\left(\exp \left\{\int_{0}^{t-1} V_{2}(Y(s)) d s\right\}\right),
$$

if $\xi$ is as in (III). Here $C_{\delta}^{\mathcal{K}}=\min _{x \in K_{\delta}} P\left(X^{\mathcal{K}}(1)=x\right)>0$ and $C_{\delta}^{\rho}=$ $\min _{x \in K_{\delta}} P_{0}\left(X^{\rho}(1)=x\right)>0$. Now proceed as in the proof of [10, Proposition 2.2.1] and then apply the Rayleigh-Ritz formula as in the proof of [10, Proposition 2.2.2]. 


\subsubsection{Proof of Proposition 2.4}

Proof We only prove the case $p=1$, the extension to general $p$ being straightforward, see also Remark 2.7. The proof of Proposition 2.4 is divided into 2 Steps.

Step 1 We first show that $\lambda_{1}(\mathcal{K})$ is bounded from above by the right-hand side of (2.10). Recall (2.12).

Claim 2.6 There is a sequence of constants $C_{t}, t>0$, with $\lim _{t \rightarrow \infty} C_{t}=\infty$ such that for all $N \in \mathbb{N}$ and $t>0$,

$$
\mathbb{E}_{\mu, 0}\left(\exp \left\{\int_{0}^{t} V_{N}\left(\xi_{s}, X^{\mathcal{K}}(s)\right) d s\right\}\right) \leqslant \mathrm{e}^{t \lambda\left(V_{N}\right)}(2 t \log t+1)^{d}+\mathrm{e}^{-C_{t} t},
$$

where $\mathbb{E}_{\mu, 0}$ denotes expectation w.r.t. the joint process $\left(\xi, X^{\mathcal{K}}\right)$ when $\xi$ is drawn from $\mu$ and $X^{\mathcal{K}}$ starts at 0 , and

$$
\lambda\left(V_{N}\right)=\sup _{\|f\|_{L^{2}(\mu \otimes m)}=1}\left\langle\left(L+\Delta^{\mathcal{K}}+V_{N}\right) f, f\right\rangle .
$$

Claim 2.6 implies the upper bound in Proposition 2.4. Indeed, via monotone convergence, for all $t>0$,

$$
\begin{aligned}
\mathbb{E}_{\mu, 0} & \left(\exp \left\{\int_{0}^{t} \xi\left(X^{\mathcal{K}}(s), s\right) d s\right\}\right) \\
& =\lim _{N \rightarrow \infty} \mathbb{E}_{\mu, 0}\left(\exp \left\{\int_{0}^{t} V_{N}\left(\xi_{s}, X^{\mathcal{K}}(s)\right) d s\right\}\right) \\
& \leq \sup _{N \in \mathbb{N}}\left\{\mathrm{e}^{t \lambda\left(V_{N}\right)}(2 t \log t+1)^{d}+e^{-C_{t} t}\right\}=\mathrm{e}^{t \sup _{N \in \mathbb{N}} \lambda\left(V_{N}\right)}(2 t \log t+1)^{d}+\mathrm{e}^{-C_{t} t} .
\end{aligned}
$$

Taking the logarithm, dividing by $t$ and letting $t \rightarrow \infty$, leads to the desired upper bound.

Before we begin the proof of Claim 2.6 we recall some facts from Gärtner and den Hollander [9]. A slight generalization of [9, Proposition 2.1] states that

$$
\begin{aligned}
\mathbb{E}_{\mu, 0}\left(\exp \left\{\int_{0}^{t} \xi\left(X^{\mathcal{K}}(s), s\right) d s\right\} u_{0}\left(X^{\mathcal{K}}(t)\right)\right) \\
=\mathrm{e}^{v t} E_{0}\left(\exp \left\{v \int_{0}^{t} w\left(X^{\mathcal{K}}(s), s\right) d s\right\} u_{0}\left(X^{\mathcal{K}}(t)\right)\right) .
\end{aligned}
$$

Here, the function $w$ is the solution of the equation

$$
\left\{\begin{array}{l}
\frac{\partial}{\partial t} w(x, t)=\Delta w(x, t)+\delta_{X} \mathcal{K}_{(t)}(x)\{w(x, t)+1\}, \quad x \in \mathbb{Z}^{d}, t \geq 0 . \\
w(x, 0)=0,
\end{array}\right.
$$


Moreover, [9, Propositions 2.2-2.3] state that there is a function $\bar{w}: \mathbb{Z}^{d} \times[0, \infty) \rightarrow$ $\mathbb{R}$ such that: (i) $w(x, t) \leq \bar{w}(0, t)$ for all $x \in \mathbb{Z}^{d}, t \geq 0$; (ii) $t \mapsto \bar{w}(0, t)$ is nondecreasing with limit

$$
\bar{w}(0)= \begin{cases}\frac{G(0)}{1-G(0)}, & \text { if } 0<1<1 / G(0), \\ \infty, & \text { otherwise. }\end{cases}
$$

We are now ready to prove Claim 2.6. We use ideas from Kipnis and Landim [13, Appendix 1.7]. Recall the uniform ellipticity assumption (1.5) on the $\mathcal{K}$-field. Thus, by standard large deviation estimates of the number of jumps of $X^{\mathcal{K}}$ and by (2.28)(2.30), there is a sequence of constants $C_{t}$ as in the statement of Claim 2.6 such that for all $t>0$ and $N \in \mathbb{N}$,

$$
\mathbb{E}_{\mu, 0}\left(\exp \left\{\int_{0}^{t} V_{N}\left(\xi_{s}, X^{\mathcal{K}}(s)\right) d s\right\} \mathbb{1}\left\{X^{\mathcal{K}}([0, t]) \subsetneq B_{R(t)}\right\}\right) \leqslant \mathrm{e}^{-C_{t} t} .
$$

Here, $B_{R(t)}$ denotes the box centered at the origin with side length $R(t)=t \log t$. We now make use of the following fact (which follows from Demuth and van Casteren [5, Theorem 2.2.5]). Let $W: \mathbb{N}_{0}^{\mathbb{Z}^{d}} \times \mathbb{Z}^{d} \rightarrow \mathbb{R}$ be a bounded function. Then $L+\Delta^{\mathcal{K}}+$ $W$ is a self-adjoint operator on $L^{2}\left(\mathbb{N}_{0}^{\mathbb{Z}^{d}} \times \mathbb{Z}^{d}, \mu \otimes m\right)$, and is the generator of the semigroup

$$
\left(P_{t}^{W} f\right)(\eta, x)=\mathbb{E}_{\eta, x}\left(\exp \left\{\int_{0}^{t} W\left(\xi_{s}, X^{\mathcal{K}}(s)\right) d s\right\} f\left(\xi_{t}, X^{\mathcal{K}}(t)\right)\right), \quad t>0 .
$$

In particular, the function $v_{t}(\eta, x)=\left(P_{t}^{V_{N}} \bar{f}\right)(\eta, x)$ with $\bar{f}(\eta, x)=\mathbb{1}\left\{x \in B_{R(t)}\right\}$ is a solution of the equation

$$
\left\{\begin{array}{l}
\frac{\partial}{\partial t} v_{t}(\eta, x)=\left(L+\Delta^{\mathcal{K}}+V_{N}\right) v_{t}(\eta, x), \\
v_{0}(\eta, x)=\bar{f}(\eta, x),
\end{array} \quad \eta \in \mathbb{N}^{\mathbb{Z}^{d}}, x \in \mathbb{Z}^{d}, t \geqslant 0 .\right.
$$

Here $V_{N}$ acts as a multiplication operator. Since $\bar{f} \in L^{2}\left(\mathbb{N}_{0}^{\mathbb{Z}^{d}} \times \mathbb{Z}^{d}, \mu \otimes m\right)$ we can write

$$
\begin{aligned}
\mathbb{E}_{\mu, 0} & \left(\exp \left\{\int_{0}^{t} V_{N}\left(\xi_{s}, X^{\mathcal{K}}(s)\right) d s\right\} \mathbb{1}\left\{X^{\mathcal{K}}([0, t]) \subset B_{R(t)}\right\}\right) \\
& \leq \mathbb{E}_{\mu, 0}\left(\exp \left\{\int_{0}^{t} V_{N}\left(\xi_{s}, X^{\mathcal{K}}(s)\right) d s\right\} \mathbb{1}\left\{X^{\mathcal{K}}(t) \in B_{R(t)}\right\}\right) \\
& \leq \int_{\mathbb{N}_{0}^{Z^{d}}} \sum_{x \in \mathbb{Z}^{d}} \mathbb{1}\left\{x \in B_{R(t)}\right\} \mathbb{E}_{\eta, x}\left(\exp \left\{\int_{0}^{t} V_{N}\left(\xi_{s}, X^{\mathcal{K}}(s)\right) d s\right\} \mathbb{1}\left\{X^{\mathcal{K}}(t) \in B_{R(t)}\right\}\right) d \mu(\eta) \\
& =\left\langle P_{t}^{V_{N}} \bar{f}, \bar{f}\right\rangle .
\end{aligned}
$$


Moreover, by (2.33), for all $t>0$,

$$
\begin{aligned}
\frac{\partial}{\partial t}\left\|P_{t}^{V_{N}} \bar{f}\right\|_{L^{2}\left(\mathbb{N}_{0}^{\mathbb{Z}^{d}} \times \mathbb{Z}^{d}, \mu \otimes m\right)}^{2} & =\int_{\mathbb{N}_{0}^{\mathbb{Z}^{d}}} \sum_{x \in \mathbb{Z}^{d}}\left[2\left(L+\Delta^{\mathcal{K}}+V_{N}\right)\left(P_{t}^{V_{N}} \bar{f}\right)(\eta, x) \times\left(P_{t}^{V_{N}} \bar{f}\right)(\eta, x)\right] d \mu(\eta) \\
& =2\left\langle\left(L+\Delta^{\mathcal{K}}+V_{N}\right) P_{t}^{V_{N}} \bar{f}, P_{t}^{V_{N}} \bar{f}\right\rangle \\
& \leqslant 2 \lambda\left(V_{N}\right)\left\|P_{t}^{V_{N}} \bar{f}\right\|_{L^{2}\left(\mathbb{N}_{0}^{\mathbb{Z}^{d}} \times \mathbb{Z}^{d}, \mu \otimes m\right)}^{2}
\end{aligned}
$$

where interchanging the derivative and the scalar product is justified by dominated convergence in combination with Lemma B.1 in the appendix section. Further note that

$$
\left\|P_{0}^{V_{N}} \bar{f}\right\|_{L^{2}\left(\mathbb{N}_{0}^{\mathbb{Z}^{d}} \times \mathbb{Z}^{d}, \mu \otimes m\right)}^{2}=\left|B_{R(t)}\right| \leqslant(2 t \log t+1)^{d},
$$

so that, by Gronwall's lemma,

$$
\left\|P_{t}^{V_{N}} \bar{f}\right\|_{L^{2}\left(\mathbb{N}_{0}^{\mathbb{Z}^{d}} \times \mathbb{Z}^{d}, \mu \otimes m\right)}^{2} \leqslant \mathrm{e}^{2 \lambda\left(V_{N}\right) t}(2 t \log t+1)^{d} .
$$

Using Cauchy-Schwarz and $\|\bar{f}\|_{L^{2}\left(\mathbb{N}_{0}^{\mathbb{Z}^{d}} \times \mathbb{Z}^{d}, \mu \otimes m\right)}^{2}=1$, we obtain that

$$
\left\langle P_{t}^{V_{N}} \bar{f}, \bar{f}\right\rangle \leq \mathrm{e}^{\lambda\left(V_{N}\right) t}(2 t \log t+1)^{d}
$$

The claim follows by combining (2.31), (2.34) and (2.38).

Step 2 It remains to show that $\lambda_{1}(\mathcal{K})$ is bounded from below by the right-hand side of (2.10). The proof follows the same line of argument as the proof of [10, Proposition 2.2.1] for $\mathcal{K} \equiv \kappa$. The details to adapt it are left to the reader since they are similar to those given in the proof of the lower bound in Section 2.4.1.

Remark 2.7 To adapt the above proof to general $p$ note that (2.30) reads in this case

$$
\bar{w}(0)= \begin{cases}\frac{p G(0)}{1-p G(0)}, & \text { if } 0<p<1 / G(0), \\ \infty, & \text { otherwise }\end{cases}
$$

\section{Annealed Lyapunov Exponents: Confinement Approximation and Lower Bound in Theorem 1.2}

In Section 3.1 we show that the annealed Lyapunov exponents for $\mathcal{K} \equiv \kappa$ do not change when the random walk in the Feynman-Kac formula (1.6) is confined to a slowly growing box (Proposition 3.1). In Section 3.2 we use this result to prove the lower bound in Theorem 1.2, i.e., $\sup \left\{\lambda_{p}(\kappa): \kappa \in \operatorname{Supp}(\mathcal{K})\right\} \leq \lambda_{p}(\mathcal{K})$. Throughout this section we assume that $u_{0}=\delta_{0}$, see Proposition 2.1 for a justification of that assumption. 


\subsection{Confinement Approximation}

Proposition 3.1 Fix $p \in \mathbb{N}$ and $\kappa>0$, and let $\xi$ be as in (I)-(III). Fix a nondecreasing function $L:[0, \infty) \rightarrow[0, \infty)$ such that $\lim _{t \rightarrow \infty} L(t)=\infty$. Then

$$
\lim _{t \rightarrow \infty} \frac{1}{p t} \log \mathbb{E}\left[E_{0}\left(\exp \left\{\int_{0}^{t} \xi\left(X^{\kappa}(s), s\right) d s\right\} \delta_{0}\left(X^{\kappa}(t)\right) \mathbb{1}\left\{X^{\kappa}[0, t] \subset B_{L(t)}(0)\right\}\right)^{p}\right]=\lambda_{p}(\kappa) .
$$

Proof We write out the proof for the dynamics (I), namely for space-time white noise. Given $p$ independent simple random walks $X_{1}^{\kappa}, X_{2}^{\kappa}, \ldots, X_{p}^{\kappa}$, write $\bar{X}^{\kappa}=$ $\left(X_{1}^{\kappa}, X_{2}^{\kappa}, \ldots, X_{p}^{\kappa}\right)$. For $0 \leq s<t<\infty$, define

$$
\begin{aligned}
\Xi^{\mathrm{STWN}}(s, t)=E_{0}^{\otimes p}\left(\exp \left\{\sum_{1 \leqslant i<j \leqslant p} \int_{0}^{t-s} \mathbb{1}\left\{X_{i}^{\kappa}(v)=X_{j}^{\kappa}(v)\right\} d v\right\}\right. \\
\left.\times \delta_{0}\left(\bar{X}^{\kappa}(t-s)\right) \mathbb{1}\left\{\bar{X}^{\kappa}[0, t-s] \subseteq B_{L(t-s)}(0)\right\}\right),
\end{aligned}
$$

where, with a slight abuse of notation, we redefine $B_{L(t)}(0)=[-L(t), L(t)]^{d p} \cap \mathbb{Z}^{d p}$. Pick $u \in[s, t]$. Using that $L$ is non-decreasing, inserting $\delta_{0}\left(\bar{X}^{\kappa}(u-s)\right)$, and using the Markov property of $\bar{X}^{\kappa}$ at time $u-s$, we see that

$$
\Xi^{\mathrm{STWN}}(s, t) \geq \Xi^{\mathrm{STWN}}(s, u) \Xi^{\mathrm{STWN}}(u, t) .
$$

Hence,

$$
\lim _{t \rightarrow \infty} \frac{1}{t} \log \Xi^{\mathrm{STWN}}(0, t)
$$

exists. Thus, in order to prove Proposition 3.1 it suffices to prove that

$$
\lim _{n \rightarrow \infty} \frac{1}{p n T} \log \Xi^{\mathrm{sTwN}}(0, n T)=\lambda_{p}(\kappa), \quad T \in(0, \infty) .
$$

Fix $T>0$. First, inserting $\mathbb{1}\left\{\bar{X}^{\kappa}[0, n T] \subseteq B_{L(n T)}(0)\right\}$ and second inserting $\delta_{0}\left(\bar{X}^{\kappa}(k T)\right), k \in\{1,2, \ldots, n-1\}$, and using the Markov property of $\bar{X}^{\kappa}$ at times $k T$ for the same set of indices, we get

$$
\begin{gathered}
E_{0}^{\otimes p}\left(\exp \left\{\sum_{1 \leqslant i<j \leqslant p} \int_{0}^{n T} \mathbb{1}\left\{X_{i}^{\kappa}(v)=X_{j}^{\kappa}(v)\right\} d v\right\} \delta_{0}\left(\bar{X}^{\kappa}(n T)\right)\right) \\
\geqslant \Xi^{\mathrm{STWN}}(0, n T) \\
\geqslant \prod_{k=1}^{n} E_{0}^{\otimes p}\left(\exp \left\{\sum_{1 \leqslant i<j \leqslant p} \int_{0}^{T} \mathbb{1}\left\{X_{i}^{\kappa}(v)=X_{j}^{\kappa}(v)\right\} d v\right\} \delta_{0}\left(\bar{X}^{\kappa}(T)\right)\right. \\
\left.\times \mathbb{1}\left\{\bar{X}^{\kappa}[0, T] \subseteq B_{L(n T)}(0)\right\}\right) .
\end{gathered}
$$


Taking the logarithm, dividing by $p n T$, and letting $n \rightarrow \infty$ followed by $T \rightarrow \infty$, we obtain

$$
\lambda_{p}(\kappa) \geq \lim _{T \rightarrow \infty} \lim _{n \rightarrow \infty} \frac{1}{p n T} \log \Xi^{\mathrm{STwN}}(0, n T) \geqslant \lambda_{p}(\kappa)
$$

which is the desired claim.

The proof for (II)-(III) works along the same lines. To use the superadditivity argument as in (3.3) and to get the inequalities in (3.6), the same techniques as in the first step of the proof of Proposition 2.1 in Appendix A may be applied.

\subsection{Proof of the Lower Bound in Theorem 1.2}

We give the proof for (I). The idea of the proof is to restrict the random walk to a box that slowly increases with time such that the $\mathcal{K}$-field is constant on this box. The existence of such a box is guaranteed by the clustering property of $\mathcal{K}$ stated in Definition 1.1. Proposition 3.1 then yields that the resulting Lyapunov exponent equals $\lambda_{p}(\kappa)$ with $\kappa$ the value of $\mathcal{K}$ on this box.

Proof The proof comes in 2 Steps.

Step 1 We first prove the lower bound in Theorem 1.2 under the assumption that $\operatorname{Supp}(\mathcal{K})=\left\{\kappa_{1}, \kappa_{2}\right\}, 0<\kappa_{1}<\kappa_{2}<\infty$. By the clustering property of $\mathcal{K}$, there is a function $L:[0, \infty) \rightarrow[0, \infty)$ with $\lim _{t \rightarrow \infty} L(t)=\infty$ such that there is a $x\left(\kappa_{l}, t\right) \in \mathbb{Z}^{d}$ with $g_{l}(t) \stackrel{\text { def }}{=}\left\|x\left(\kappa_{l}, t\right)\right\| \in o(t)$ such that $\mathcal{K}(x, y)=\kappa_{l}$ for all edges $(x, y) \in B_{L(t)}\left(x\left(\kappa_{l}, t\right)\right), l \in\{1,2\}$. We fix $l \in\{1,2\}$ and, as in the proof of Proposition 3.1 , denote by $\bar{X}^{\mathcal{K}}$ the $\mathbb{Z}^{d p}$-valued process $\left(X_{1}^{\mathcal{K}}, \ldots, X_{p}^{\mathcal{K}}\right)$. We further denote by $\bar{x}\left(\kappa_{l}, t\right) \in \mathbb{Z}^{d p}$ the vertex given by $\left(x_{1}\left(\kappa_{l}, t\right), \ldots, x_{p}\left(\kappa_{l}, t\right)\right)$, where $x_{i}\left(\kappa_{l}, t\right)=x\left(\kappa_{l}, t\right)$ for each $i \in\{1, \ldots, p\}$. An application of the Markov property of $\bar{X}^{\mathcal{K}}$ at times $g_{l}(t)$ and $t-g_{l}(t)$ yields

$$
\begin{aligned}
& E_{0}^{\otimes p}\left(\exp \left\{\sum_{1 \leqslant i<j \leqslant p} \int_{0}^{t} \mathbb{1}\left\{X_{i}^{\mathcal{K}}(s)=X_{j}^{\mathcal{K}}(s)\right\} d s\right\} \delta_{0}\left(\bar{X}^{\mathcal{K}}(t)\right)\right) \\
& \geqslant E_{0}^{\otimes p}\left(\exp \left\{\sum_{1 \leqslant i<j \leqslant p} \int_{0}^{g_{l}(t)} \mathbb{1}\left\{X_{i}^{\mathcal{K}}(s)=X_{j}^{\mathcal{K}}(s)\right\} d s\right\} \delta_{\bar{x}\left(\kappa_{l}, t\right)}\left(\bar{X}^{\mathcal{K}}\left(g_{l}(t)\right)\right)\right) \\
& \quad \times E_{\bar{x}\left(\kappa_{l}, t\right)}^{\otimes p}\left(\exp \left\{\sum_{1 \leqslant i<j \leqslant p} \int_{0}^{t-2 g_{l}(t)} \mathbb{1}\left\{X_{i}^{\mathcal{K}}(s)=X_{j}^{\mathcal{K}}(s)\right\} d s\right\} \delta_{\bar{x}\left(\kappa_{l}, t\right)}\left(\bar{X}^{\mathcal{K}}\left(t-2 g_{l}(t)\right)\right)\right) \\
& \quad \times E_{\bar{x}\left(\kappa_{l}, t\right)}^{\otimes p}\left(\exp \left\{\sum_{1 \leqslant i<j \leqslant p} \int_{0}^{g_{l}(t)} \mathbb{1}\left\{X_{i}^{\mathcal{K}}(s)=X_{j}^{\mathcal{K}}(s)\right\} d s\right\} \delta_{0}\left(\bar{X}^{\mathcal{K}}\left(g_{l}(t)\right)\right)\right) \\
& \quad \stackrel{\operatorname{def}}{=} U_{1}(t) \times U_{2}(t) \times U_{3}(t) .
\end{aligned}
$$


Note that

$$
U_{1}(t) \geqslant P_{0}\left(\bar{X}^{\mathcal{K}}\left(g_{l}(t)\right)=\bar{x}\left(\kappa_{l}, t\right)\right),
$$

which is bounded from below by

$$
\left(\frac{\kappa_{1}}{2 d \kappa_{2}}\right)^{g_{l}(t)} \mathrm{e}^{-2 d \kappa_{2} g_{l}(t)} \frac{\left(2 d \kappa_{1} g_{l}(t)\right)^{g_{l}(t)}}{g_{l}(t) !},
$$

so that $\lim _{t \rightarrow \infty} \frac{1}{t} \log U_{1}(t)=0$. The same reasoning shows that also $\lim _{t \rightarrow \infty} \frac{1}{t} \log U_{3}(t)=0$. To control $U_{2}$, we use the lower bound

$$
\begin{aligned}
U_{2}(t) \geqslant E_{\bar{x}\left(\kappa_{l}, t\right)}^{\otimes p}(\exp & \left\{\sum_{1 \leqslant i<j \leqslant p} \int_{0}^{t-2 g_{l}(t)} \mathbb{1}\left\{X_{i}^{\mathcal{K}}(s)=X_{j}^{\mathcal{K}}(s)\right\} d s\right\} \delta_{\bar{x}\left(\kappa_{l}, t\right)}\left(\bar{X}^{\mathcal{K}}\left(t-2 g_{l}(t)\right)\right) \\
& \left.\times \prod_{i=1}^{p} \mathbb{1}\left\{X_{i}^{\mathcal{K}}\left[0, t-2 g_{l}(t)\right] \subseteq B_{L(t)-1}\left(x\left(\kappa_{l}, t\right)\right)\right\}\right) .
\end{aligned}
$$

Note that for each $i \in\{1, \ldots, p\}$ the random walk $X_{i}^{\mathcal{K}}$ on the event $\left\{X_{i}^{\mathcal{K}}[0, t] \subseteq\right.$ $\left.B_{L(t)-1}\left(x\left(\kappa_{l}, t\right)\right)\right\}$ is distributed as a random walk with diffusion constant $\kappa_{l}$ confined to stay in this box. Hence, by the shift invariance of $\bar{X}^{\kappa}$ in space and Proposition 3.1,

$$
\begin{aligned}
U_{2}(t) \geqslant & E_{0}^{\otimes p}\left(\exp \left\{\sum_{1 \leqslant i<j \leqslant p} \int_{0}^{t-2 g_{l}(t)} \mathbb{1}\left\{X_{i}^{\kappa_{l}}(s)=X_{j}^{\kappa_{l}}(s)\right\} d s\right\} \delta_{0}\left(\bar{X}^{\kappa_{l}}\left(t-2 g_{l}(t)\right)\right)\right. \\
& \left.\times \prod_{i=1}^{p} \mathbb{1}\left\{X_{i}^{\kappa_{l}}\left[0, t-2 g_{l}(t)\right] \subseteq B_{L(t)-1}(0)\right\}\right) \\
\geqslant & \mathrm{e}^{\lambda_{p}\left(\kappa_{l}\right)\left(t-2 g_{l}(t)\right) p+o(t)} .
\end{aligned}
$$

Finally, (3.8-3.12) yield that

$$
\lambda_{p}(\mathcal{K}) \geqslant \max \left\{\lambda_{p}\left(\kappa_{1}\right), \lambda_{p}\left(\kappa_{2}\right)\right\},
$$

which settles Theorem 1.2 for the case where $\operatorname{Supp}(\mathcal{K})=\left\{\kappa_{1}, \kappa_{2}\right\}, \kappa_{1}, \kappa_{2} \in(0, \infty)$.

Step 2 We next prove Theorem 1.2 for the general case by reducing it to the setting of Step 1. Recall (2.20). Fix $n \in \mathbb{N}$. Given a realization of $\mathcal{K}$, we define a discretization $\mathcal{K}_{n}$ of $\mathcal{K}$ by putting, for each $x, y \in \mathbb{Z}^{d}$,

$$
\begin{aligned}
& \mathcal{K}_{n}(x, y) \\
& = \begin{cases}\kappa_{*}+(j-1) \frac{\left(\kappa^{*}-\kappa_{*}\right)}{n}, & \text { if } \kappa_{*}+(j-1) \frac{\left(\kappa^{*}-\kappa_{*}\right)}{n} \leqslant \mathcal{K}(x, y)<\kappa_{*}+j \frac{\left(\kappa^{*}-\kappa_{*}\right)}{n}, 1 \leqslant j \leqslant n, \\
\kappa^{*}, & \text { if } \mathcal{K}(x, y)=\kappa^{*} .\end{cases}
\end{aligned}
$$


A slight adaptation of Step 1 yields

$$
\lambda_{p}\left(\mathcal{K}_{n}\right) \geqslant \max \left\{\lambda_{p}(\kappa), \kappa \in \operatorname{Supp}\left(\mathcal{K}_{n}\right) \backslash\left\{\kappa^{*}\right\}\right\} .
$$

Here, the restriction to the set $\operatorname{Supp}\left(\mathcal{K}_{n}\right) \backslash\left\{\kappa^{*}\right\}$ comes from the fact that $\tilde{\mathbb{P}}(\mathcal{K}(x, y)=$ $\left.\kappa^{*}\right)=0$ is possible, e.g. when the distribution of $\mathcal{K}$ is continuous. By Carmona and Molchanov [3, Proposition III.2.7], $\kappa \mapsto \lambda_{p}(\kappa)$ is continuous, hence the right-hand side of (3.15) converges to $\sup \left\{\lambda_{p}(\kappa), \kappa \in \operatorname{Supp}(\mathcal{K})\right\}$ as $n \rightarrow \infty$. Hence it suffices to show that $\limsup _{n \rightarrow \infty} \lambda_{p}\left(\mathcal{K}_{n}\right) \leq \lambda_{p}(\mathcal{K})$.

To do so we borrow ideas from the proof of [11, Theorem 1.2(i)]. First we introduce the notation $\tilde{\mathcal{K}}(x)=\sum_{y \in \mathbb{Z}^{d}} \mathcal{K}(x, y), x \in \mathbb{Z}^{d}$, and we define $\tilde{\mathcal{K}}_{n}$ in a similar fashion. An application of Girsanov's formula yields that (see König, Salvi and Wolff [14, Lemma 2.1])

$$
\begin{aligned}
& E_{0}^{\otimes p}\left(\exp \left\{\sum_{1 \leqslant i<j \leqslant p} \int_{0}^{t} \mathbb{1}\left\{X_{i}^{\mathcal{K}_{n}}(s)=X_{j}^{\mathcal{K}_{n}}(s)\right\} d s\right\} \delta_{0}\left(\bar{X}^{\mathcal{K}_{n}}(t)\right)\right) \\
& =E_{0}^{\otimes p}\left(\exp \left\{\sum_{1 \leqslant i<j \leqslant p} \int_{0}^{t} \mathbb{1}\left\{X_{i}^{\mathcal{K}}(s)=X_{j}^{\mathcal{K}}(s)\right\} d s\right\} \delta_{0}\left(\bar{X}^{\mathcal{K}}(t)\right)\right. \\
& \times \exp \left\{\sum_{1 \leqslant i \leqslant p} \sum_{l=1}^{N\left(X_{i}^{\mathcal{K}} ; t\right)} \log \left[\frac{\mathcal{K}_{n}\left(X_{i}^{\mathcal{K}}\left(S_{l-1}\right), X_{i}^{\mathcal{K}}\left(S_{l}\right)\right)}{\mathcal{K}\left(X_{i}^{\mathcal{K}}\left(S_{l-1}\right), X_{i}^{\mathcal{K}}\left(S_{l}\right)\right)}\right]\right. \\
& \left.\left.-\int_{0}^{t}\left[\tilde{\mathcal{K}}_{n}\left(X_{i}^{\mathcal{K}}(s)\right)-\tilde{\mathcal{K}}\left(X_{i}^{\mathcal{K}}(s)\right)\right] d s\right\}\right),
\end{aligned}
$$

where $N\left(X^{\mathcal{K}} ; t\right)$ denotes the number of jumps of the random walk $X^{\mathcal{K}}$ with generator $\Delta^{\mathcal{K}}$ up to time $t$. Note that $\frac{\mathcal{K}_{n}(x, y)}{\mathcal{K}(x, y)} \leq 1$ for all $x \sim y \in \mathbb{Z}^{d}$ and that $-\int_{0}^{t}\left[\tilde{\mathcal{K}}_{n}\left(X^{\mathcal{K}}(s)\right)-\tilde{\mathcal{K}}\left(X^{\mathcal{K}}(s)\right)\right] d s \leq 2 d t / n$. Hence, the right-hand side of (3.16) is bounded from above by

$$
E_{0}^{\otimes p}\left(\exp \left\{\sum_{1 \leqslant i<j \leqslant p} \int_{0}^{t} \mathbb{1}\left\{X_{i}^{\mathcal{K}}(s)=X_{j}^{\mathcal{K}}(s)\right\} d s\right\} \delta_{0}\left(\bar{X}^{\mathcal{K}}(t)\right)\right) \mathrm{e}^{2 d t / n} .
$$

Consequently, (3.16) and (3.17) show that $\limsup _{n \rightarrow \infty} \lambda_{p}\left(\mathcal{K}_{n}\right) \leq \lambda_{p}(\mathcal{K})$. This finishes the proof. The proof for (II) and (III) is the same as above, with the additional restriction that $0<p<1 / G(0)$ for (IIb). To get the inequality in (3.8) we use the techniques in the first step of the proof of Proposition 2.1 in Appendix A. By Castell, Gün and Maillard [4, Theorem 1.1(ii)] and Gärtner and den Hollander [9, Theorem 1.5], $\kappa \mapsto \lambda_{p}(\kappa)$ is continuous for (II), which allows us to take the limit on the right-hand side of (3.15). The continuity of $\kappa \mapsto \lambda_{p}(\kappa)$ for (III) follows from Proposition 2.5, which still holds when $\kappa$ is deterministic. Indeed, the variational formula in Proposition 2.5 shows that $\kappa \mapsto \lambda_{p}(\kappa)$ is convex. Since $\xi$ is bounded for (III), so is $\kappa \mapsto \lambda_{p}(\kappa)$, which yields the desired continuity. To obtain the result for (IIb) with $p \geq 1 / G(0)$, for which $\lambda_{p}(\kappa)=\infty$ for all $\kappa \geq 0$, we note that averaging $u(0, t)^{p}$ 
first with respect to the trajectories $Y_{j}^{y}$ present in the definition of $\xi$, then with respect to the Poisson field $\left(N_{y}\right)_{y \in \mathbb{Z}^{d}}$ and using standard Feynman-Kac identities, an adaption of the proof of [9, Proposition 2.1] yields the estimate

$$
\begin{aligned}
\mathbb{E}\left[u(0, t)^{p}\right] & \geqslant \mathbb{E}\left[E_{0}\left(\exp \left\{\int_{0}^{t} \xi\left(X^{\mathcal{K}}(s), t-s\right) d s\right\} \mathbb{1}\left\{X^{\mathcal{K}}(s)=0 \text { for all } s \in[0, t]\right\}\right)^{p}\right] \\
& \geqslant \exp \left\{-p t \sum_{\|x\|=1} \mathcal{K}(0, x)+p v t\right\} \exp \left\{p \int_{0}^{t} \bar{w}(0, s) d s\right\},
\end{aligned}
$$

where $\bar{w}$ solves the equation

$$
\left\{\begin{array}{l}
\frac{\partial}{\partial t} \bar{w}(x, t)=\Delta \bar{w}(x, t)+\delta_{0}(x)[\bar{w}(x, t)+1], \quad x \in \mathbb{Z}^{d}, t \geqslant 0 . \\
w(x, 0)=0,
\end{array}\right.
$$

To conclude it suffices to note that by [9, Proposition 2.3] (with the notation $r_{d}=$ $1 / G(0)), t \mapsto \bar{w}(0, t)$ is non-decreasing with $\lim _{t \rightarrow \infty} \bar{w}(0, t)=\infty$.

\section{Quenched Lyapunov Exponent: Confinement Approximation and Lower Bound}

In Section 4.1 we show that a confinement approximation holds for $\mathcal{K} \equiv \kappa$. In Section 4.2 we use this result to prove Theorem 1.3.

\subsection{Confinement Approximation}

Proposition 4.1 Let $L:[0, \infty) \rightarrow[0, \infty)$ be non-decreasing with $\lim _{t \rightarrow \infty} L(t)=$ $\infty$. Then $\mathbb{P}$-a.s. and in $\mathbb{P}$-mean,

$$
\lim _{t \rightarrow \infty} \frac{1}{t} \log E_{0}\left(\exp \left\{\int_{0}^{t} \xi\left(X^{\kappa}(s), s\right) d s\right\} \delta_{0}\left(X^{\kappa}(t)\right) \mathbb{1}\left\{X^{\kappa}[0, t] \subseteq B_{L(t)}(0)\right\}\right)=\lambda_{0}(\kappa) .
$$

Proof For $0 \leq s \leq t<\infty$, define

$$
\Xi(s, t)=E_{0}\left(\exp \left\{\int_{0}^{t-s} \xi\left(X^{\kappa}(v), s+v\right) d v\right\} \delta_{0}\left(X^{\kappa}(t-s)\right) \mathbb{1}\left\{X^{\kappa}[0, t-s] \subseteq B_{L(t-s)}(0)\right\}\right) .
$$


Pick $u \in[s, t]$. Using that $L$ is non-decreasing and inserting $\delta_{0}\left(X^{\kappa}(u-s)\right)$ under the expectation in (4.2), we obtain

$$
\begin{aligned}
\Xi(s, t) \geqslant & E_{0}\left(\exp \left\{\int_{0}^{u-s} \xi\left(X^{\kappa}(v), s+v\right) d v\right\} \delta_{0}\left(X^{\kappa}(u-s)\right) \mathbb{1}\left\{X^{\kappa}[0, u-s] \subseteq B_{L(u-s)}(0)\right\}\right. \\
& \left.\times \exp \left\{\int_{u-s}^{t-s} \xi\left(X^{\kappa}(v), s+v\right) d v\right\} \delta_{0}\left(X^{\kappa}(t-s)\right) \mathbb{1}\left\{X^{\kappa}[u-s, t-s] \subseteq B_{L(t-u)}(0)\right\}\right) .
\end{aligned}
$$

Applying the Markov property of $X^{\kappa}$ at time $u-s$, we get

$$
\Xi(s, t) \geqslant \Xi(s, u) \Xi(u, t), \quad 0 \leqslant s \leqslant u \leqslant t<\infty .
$$

Since $\xi$ is stationary and ergodic, and the law of $\{\Xi(u+s, u+t): 0 \leq s \leq t<\infty\}$ is the same for all $u \geq 0$, it follows from Kingman's superadditive ergodic theorem [12] that

$$
\lim _{t \rightarrow \infty} \frac{1}{t} \log \Xi(0, t) \text { exists } \mathbb{P} \text {-a.s. and in } \mathbb{P} \text {-mean, and is non-random. }
$$

Thus, in order to prove (4.1), it suffices to show that

$$
\lim _{n \rightarrow \infty} \frac{1}{n T} \log \Xi(0, n T)=\lambda_{0}(\kappa), \quad T \in(0, \infty) .
$$

Inserting $\mathbb{1}\left\{X^{\kappa}[0, n T] \subset B_{L(n T)}(0)\right\}$ and $\delta_{0}\left(X^{\kappa}(k T)\right), k \in\{1,2, \ldots, n-1\}$, and using the Markov property of $X^{\kappa}$ at times $k T$ for the same set of indices, we get

$$
\begin{aligned}
& E_{0}\left(\exp \left\{\int_{0}^{n T} \xi\left(X^{\kappa}(s), s\right) d s\right\} \delta_{0}\left(X^{\kappa}(n T)\right)\right) \\
& \geqslant \Xi(0, n T) \\
& \geqslant \prod_{i=1}^{n} E_{0}\left(\exp \left\{\int_{0}^{T} \xi\left(X^{\kappa}(s),(i-1) T+s\right) d s\right\} \delta_{0}\left(X^{\kappa}(T)\right) \mathbb{1}\left\{X^{\kappa}[0, T] \subseteq B_{L(n T)}(0)\right\}\right) .
\end{aligned}
$$

Using that $\xi$ is invariant under time shifts, we get

$$
\begin{aligned}
\frac{1}{n T} & \mathbb{E}\left[\log E_{0}\left(\exp \left\{\int_{0}^{n T} \xi\left(X^{\kappa}(s), s\right) d s\right\} \delta_{0}\left(X^{\kappa}(n T)\right)\right)\right] \\
& \geqslant \frac{1}{n T} \mathbb{E}[\log \Xi(0, n T)] \\
& \geqslant \frac{1}{T} \mathbb{E}\left[\log E_{0}\left(\exp \left\{\int_{0}^{T} \xi\left(X^{\kappa}(s), s\right) d s\right\} \delta_{0}\left(X^{\kappa}(T)\right) \mathbb{1}\left\{X^{\kappa}[0, T] \subseteq B_{L(n T)}(0)\right\}\right)\right] .
\end{aligned}
$$


Letting $n \rightarrow \infty$ followed by $T \rightarrow \infty$, using the $L^{1}$-convergence in (4.5), and recalling that $u_{0}=\delta_{0}$, we arrive at the sandwich

$$
\lambda_{0}(\kappa) \geqslant \lim _{T \rightarrow \infty} \lim _{n \rightarrow \infty} \frac{1}{n T} \log \Xi(0, n T) \geqslant \lambda_{0}(\kappa) .
$$

The convergence of the rightmost term in (4.8) to the rightmost term in (4.5) can be shown by a direct comparison between these two terms using condition (4) for $\xi$.

\subsection{Proof of Theorem 1.3}

With the help of Proposition 4.1 we can now give the proof of Theorem 1.3.

Proof The proof comes in 3 Steps.

Step 1 The proof of the existence in an almost sure sense and in the $L^{1}$-sense of the quenched Lyapunov exponent follows along the lines of the proof of [11, Theorem 1.1].

Step 2 We first prove (1.21) under the assumption $\operatorname{Supp}(\mathcal{K})=\left\{\kappa_{1}, \kappa_{2}\right\}, \kappa_{1}, \kappa_{2} \in$ $(0, \infty)$. By the clustering property of $\mathcal{K}$, there exists a function $L:[0, \infty) \rightarrow[0, \infty)$ with $\lim _{t \rightarrow \infty} L(t)=\infty$ such that there is an $x\left(\kappa_{i}, t\right) \in \mathbb{Z}^{d}$ with $g_{i}(t)=\left\|x\left(\kappa_{i}, t\right)\right\| \in$ $o(t)$ such that $\mathcal{K}(x, y)=\kappa_{i}$ for all $(x, y) \in B_{L(t)}\left(x\left(\kappa_{i}, t\right)\right), i \in\{1,2\}$. We fix $i \in\{1,2\}$. An application of the Markov property of the random walk at times $g_{i}(t)$ and $t-g_{i}(t)$ yields

$$
\begin{aligned}
& E_{0}\left(\exp \left\{\int_{0}^{t} \xi\left(X^{\mathcal{K}}(s), s\right) d s\right\} \delta_{0}\left(X^{\mathcal{K}}(t)\right)\right) \\
& \geqslant E_{0}\left(\exp \left\{\int_{0}^{g_{i}(t)} \xi\left(X^{\mathcal{K}}(s), s\right) d s\right\} \delta_{x\left(\kappa_{i}, t\right)}\left(X^{\mathcal{K}}\left(g_{i}(t)\right)\right)\right) \\
& \quad \times E_{x\left(\kappa_{i}, t\right)}\left(\exp \left\{\int_{0}^{t-2 g_{i}(t)} \xi\left(X^{\mathcal{K}}(s), s+g_{i}(t)\right) d s\right\} \delta_{x\left(\kappa_{i}, t\right)}\left(X^{\mathcal{K}}\left(t-2 g_{i}(t)\right)\right)\right) \\
& \quad \times E_{x\left(\kappa_{i}, t\right)}\left(\exp \left\{\int_{0}^{g_{i}(t)} \xi\left(X^{\mathcal{K}}(s), s+t-g_{i}(t)\right) d s\right\} \delta_{0}\left(X^{\mathcal{K}}\left(g_{i}(t)\right)\right)\right) \\
& \quad \stackrel{\operatorname{def}}{=} U_{1}(t) \times U_{2}(t) \times U_{3}(t) .
\end{aligned}
$$


Further note that, by Jensen's inequality,

$$
\begin{aligned}
& \mathbb{E}\left[\log U_{1}(t)\right] \\
& \quad \geqslant \mathbb{E}\left[E_{0}\left(\int_{0}^{g_{i}(t)} \xi\left(X^{\mathcal{K}}(s), s\right) d s \mid X^{\mathcal{K}}\left(g_{i}(t)\right)=x\left(\kappa_{i}, t\right)\right)\right]+\log P_{0}\left(X^{\mathcal{K}}\left(g_{i}(t)\right)=x\left(\kappa_{i}, t\right)\right) \\
& \quad=E_{0}\left[\int_{0}^{g_{i}(t)} \mathbb{E}\left(\xi\left(X^{\mathcal{K}}(s), s\right)\right) d s \mid X^{\mathcal{K}}\left(g_{i}(t)\right)=x\left(\kappa_{i}, t\right)\right]+\log P_{0}\left(X^{\mathcal{K}}\left(g_{i}(t)\right)=x\left(\kappa_{i}, t\right)\right) \\
& \quad=\mathbb{E}(\xi(0,0)) g_{i}(t)+\log P_{0}\left(X^{\mathcal{K}}\left(g_{i}(t)\right)=x\left(\kappa_{i}, t\right)\right),
\end{aligned}
$$

where the interchange of the expectations is justified because

$$
\mathbb{E}\left[E_{0}\left(\int_{0}^{g_{i}(t)}\left|\xi\left(X^{\mathcal{K}}(s), s\right)\right| d s \mid X^{\mathcal{K}}\left(g_{i}(t)\right)=x\left(\kappa_{i}, t\right)\right)\right]=\mathbb{E}(|\xi(0,0)|) g_{i}(t)<\infty
$$

A similar computation yields the same lower bound for $\mathbb{E}\left[\log U_{3}(t)\right]$. Note that the lower bounds are sublinear in $t$. To control $U_{2}$, note that $X^{\mathcal{K}}$ restricted to the event $\left\{X^{\mathcal{K}}[0, t] \subset B_{L(t)-1}\left(x\left(\kappa_{i}, t\right)\right)\right\}$ is distributed as a random walk with diffusion constant $\kappa_{i}$ confined to stay in this box. Hence

$$
\begin{gathered}
U_{2}(t) \geqslant E_{x\left(\kappa_{i}, t\right)}\left(\exp \left\{\int_{0}^{t-2 g_{i}(t)} \xi\left(X^{\kappa_{i}}(s), s+g_{i}(t)\right) d s\right\} \delta_{x\left(\kappa_{i}, t\right)}\left(X^{\kappa_{i}}\left(t-2 g_{i}(t)\right)\right)\right. \\
\left.\times \mathbb{1}\left\{X^{\kappa_{i}}\left[0, t-2 g_{i}(t)\right] \subset B_{L(t)-1}\left(x\left(\kappa_{i}, t\right)\right)\right\}\right),
\end{gathered}
$$

so that, by the space-time shift invariance of $\xi$ and Proposition 4.1,

$$
\begin{aligned}
\mathbb{E} \log U_{2}(t) \geqslant \mathbb{E} \log E_{0}\left(\exp \left\{\int_{0}^{t-2 g_{i}(t)} \xi\left(X^{\kappa_{i}}(s), s\right) d s\right\} \delta_{0}\left(X^{\kappa_{i}}\left(t-2 g_{i}(t)\right)\right)\right. \\
\left.\times \mathbb{1}\left\{X^{\kappa_{i}}\left[0, t-2 g_{i}(t)\right] \subset B_{L(t)-1}(0)\right\}\right) \\
\geqslant e^{\lambda_{0}\left(\kappa_{i}\right)\left(t-2 g_{i}(t)\right)+o(t)} .
\end{aligned}
$$

Since, by the first step of the proof, we have the representation

$$
\lambda_{0}(\mathcal{K})=\lim _{t \rightarrow \infty} \frac{1}{t} \mathbb{E}(\log u(0, t)),
$$

(4.10-4.14) yield

$$
\lambda_{0}(\mathcal{K}) \geqslant \max \left\{\lambda_{0}\left(\kappa_{1}\right), \lambda_{0}\left(\kappa_{2}\right)\right\},
$$

which settles the claim for the case $\operatorname{Supp}(\mathcal{K})=\left\{\kappa_{1}, \kappa_{2}\right\}, \kappa_{1}, \kappa_{2} \in(0, \infty)$. 
Step 3 The strategy to extend the proof to the general case works similarly as in the second step of the proof of Theorem 1.2 in Section 3.2. However, since we do not know whether $\kappa \mapsto \lambda_{0}(\kappa)$ is continuous, some modifications are needed (see [11, Theorem 1.2(i)], where conditions are provided under which the quenched Lyapunov exponent $\lambda_{0}(\kappa)$ is Lipschitz continuous outside any neighbourhood of zero). Fix $n \in$ $\mathbb{N}$ and given a realisation of $\mathcal{K}$ define a discretization $\mathcal{K}_{n}$ of $\mathcal{K}$ as in the second step of the proof of Theorem 1.2. An adaptation of Step 1 yields

$$
\lambda_{0}\left(\mathcal{K}_{n}\right) \geqslant \max \left\{\lambda_{0}(\kappa), \kappa \in \operatorname{Supp}\left(\mathcal{K}_{n}\right) \backslash\left\{\kappa^{*}\right\}\right\}
$$

To continue, we claim that $\kappa \mapsto \lambda_{0}(\kappa)$ is lower semi-continuous on $(0, \infty)$. Indeed, fix $t>0$ and $\kappa \in(0, \infty)$, as well as a sequence $\left(\kappa_{n}\right)_{n \in \mathbb{N}}$ such that $\kappa_{n} \rightarrow \kappa$ as $n \rightarrow \infty$. An application of Girsanov's formula yields that

$$
\begin{aligned}
& u\left(0, t ; \kappa_{n}\right) \\
& =E_{0}\left(\exp \left\{\int_{0}^{t} \xi\left(X^{\kappa_{n}}(s), s\right) d s\right\} \delta_{0}\left(X^{\kappa_{n}}(t)\right)\right) \\
& =E_{0}\left(\exp \left\{\int_{0}^{t} \xi\left(X^{\kappa}(s), s\right) d s\right\} \delta_{0}\left(X^{\kappa}(t)\right) \exp \left\{N\left(X^{\kappa} ; t\right) \log \left[\frac{\kappa_{n}}{\kappa}\right]-2 d t\left[\kappa_{n}-\kappa\right]\right\}\right) .
\end{aligned}
$$

Hence, from Fatou's lemma we get that $\liminf _{n \rightarrow \infty} u\left(0, t ; \kappa_{n}\right) \geq u(0, t ; \kappa)$. This shows that $\kappa \mapsto u(0, t ; \kappa)$ is lower semi-continuous for all $t>0$. Using that

$$
\lambda_{0}(\kappa)=\sup _{t>0} \frac{1}{t} \log u(0, t ; \kappa)
$$

(see the proof of [11, Theorem 1.1]), we get the claim by using that suprema of lower semi-continuous functions are lower semi-continuous.

To proceed, let $M=\sup \left\{\lambda_{0}(\kappa), \kappa \in \operatorname{Supp}(\mathcal{K})\right\}$. We claim that the liminf of the right-hand side of (4.17) is bounded from below by $M$. We distinguish between two cases. If $M=\infty$, then for each $R>0$ there is $\kappa_{R} \in \operatorname{Supp}(\mathcal{K}) \operatorname{such}$ that $\lambda_{0}\left(\kappa_{R}\right) \geq R$. Since $\kappa \mapsto \lambda_{0}(\kappa)$ is lower semi-continuous, for any $\varepsilon>0$ there is a neighborhood $\mathcal{U}_{R}$ of $\kappa_{R}$ such that $\lambda_{0}(\kappa) \geq \lambda_{0}\left(\kappa_{R}\right)-\varepsilon$ for all $\kappa \in \mathcal{U}_{R}$. Hence, for all $R \geq 0$ and $\varepsilon>0$, we obtain

$$
\liminf _{n \rightarrow \infty} \max \left\{\lambda_{0}(\kappa), \kappa \in \operatorname{Supp}\left(\mathcal{K}_{n}\right) \backslash\left\{\kappa^{*}\right\}\right\} \geqslant R-\varepsilon .
$$

From this we get the claim by letting $R \rightarrow \infty$. The case $M<\infty$, may be treated similarly. It only remains to show that $\lim \sup _{n \rightarrow \infty} \lambda_{0}\left(\mathcal{K}_{n}\right) \leq \lambda_{0}(\mathcal{K})$. But this works verbatim as in the second step of the proof of Theorem 1.2. 


\section{Quenched Lyapunov Exponent: Failure of Upper Bound}

In this section we provide an example where the upper bound fails for a decorated version of $\mathbb{Z}^{d}$, namely, we show that there is a choice of $\mathcal{K}$ for which

$$
\lambda_{0}(\mathcal{K})>\sup \left\{\lambda_{0}(\kappa): \kappa \in \operatorname{Supp}(\mathcal{K})\right\} .
$$

Let $(V, \mathcal{E})$ denote the usual graph associated with $\mathbb{Z}^{d}$, i.e., $V=\mathbb{Z}^{d}$ and $\mathcal{E}=$ $\{e(x, y): x, y \in V, x \sim y\}$ is the set of edges connecting nearest-neighbour vertices of $V$. We consider $\left(V^{\star}, \mathcal{E}^{\star}\right)$, a decorated version of $(V, \mathcal{E})$, where $V^{\star}=V$ but

$$
\mathcal{E}^{\star}=\left\{\left(\mathrm{e}_{1}(x, y), \mathrm{e}_{2}(x, y)\right): x, y \in V, x \sim y\right\},
$$

i.e., we draw two edges rather than one, say red and green, between every pair of nearest-neighbour vertices of $\mathbb{Z}^{d}$.

Pick any $\mathcal{K}$ on $\mathcal{E}^{\star}$ that has the alternating cluster property, i.e., there exist boxes $B_{L(t)}$, with $\lim _{t \rightarrow \infty} L(t)=\infty$, on which all red edges have value $\kappa_{1}$ and all green edges have value $\kappa_{2}$. For such $\mathcal{K}$, by the confinement approximation of Proposition 4.1, we have

$$
\lambda_{0}(\mathcal{K}) \geqslant \lambda_{0}\left(\mathcal{K} \equiv\left(\kappa_{1}, \kappa_{2}\right)^{\mathcal{E}}\right)=\lambda_{0}\left(\kappa_{1}+\kappa_{2}\right),
$$

where $\left(\kappa_{1}, \kappa_{2}\right)^{\mathcal{E}}$ means that all red edges take value $\kappa_{1}$ and all green edges take value $\kappa_{2}$. In [11] we exhibited a class of dynamic random environments $\xi$ for which

$$
\begin{aligned}
& \kappa \mapsto \lambda_{0}(\kappa) \text { is continuous on }[0, \infty), \\
& \lambda_{0}(\kappa)>\mathbb{E}(\xi(0,0)) \forall \kappa \in(0, \infty), \\
& \lim _{\kappa \rightarrow \infty} \lambda_{0}(\kappa)=\lambda_{0}(0)=\mathbb{E}(\xi(0,0)) .
\end{aligned}
$$

In particular, $\kappa \mapsto \lambda_{0}(\kappa)$ is not monotone on $[0, \infty)$. Hence there exist $\bar{\kappa}_{1}, \bar{\kappa}_{2} \in$ $(0, \infty)$ such that

$$
\lambda_{0}\left(\frac{\bar{\kappa}_{1}+\bar{\kappa}_{2}}{2}\right)>\max \left\{\lambda_{0}\left(\bar{\kappa}_{1}\right), \lambda_{0}\left(\bar{\kappa}_{2}\right)\right\} .
$$

Picking $\kappa_{1}=\bar{\kappa}_{1} / 2$ and $\kappa_{2}=\bar{\kappa}_{2} / 2$, we get

$$
\lambda_{0}\left(\mathcal{K} \equiv\left(\kappa_{1}, \kappa_{2}\right)^{\mathcal{E}}\right)>\max \left\{\lambda_{0}\left(\mathcal{K} \equiv\left(\kappa_{1}, \kappa_{1}\right)^{\mathcal{E}}\right), \lambda_{0}\left(\mathcal{K} \equiv\left(\kappa_{2}, \kappa_{2}\right)^{\mathcal{E}}\right)\right\} .
$$

Combining (5.3) and (5.6), we arrive at (5.1).

The above counterexample does not apply to $\lambda_{0}(\mathcal{K})$ on $(V, \mathcal{E})$. Nevertheless, since all previous theory developed for $\lambda_{0}(\mathcal{K})$ on $(V, \mathcal{E})$ carries over to $\left(V^{\star}, \mathcal{E}^{\star}\right)$, the above example shows that there is little hope for the upper bound to hold for $\mathbb{Z}^{d}$.

Acknowledgments The authors were supported by ERC Advanced Grant 267356 VARIS of FdH. DE was also supported by ERC Consolidator Grant 615897 CRITICAL of Martin Hairer. GM is grateful to the Mathematical Institute of Leiden University for hospitality during sabbatical leaves in May-July 2014 and in June-July 2015.

Open Access This article is distributed under the terms of the Creative Commons Attribution 4.0 International License (http://creativecommons.org/licenses/by/4.0/), which permits unrestricted use, distribution, and reproduction in any medium, provided you give appropriate credit to the original author(s) and the source, provide a link to the Creative Commons license, and indicate if changes were made. 


\section{Appendix}

\section{A Restriction to a Localized Initial Condition}

In this appendix we prove Proposition 2.1. The proof is somewhat long and technical, but the flexibility in the choice of initial condition is important. The proof is an adaptation of the proof of Drewitz, Gärtner, Ramirez and Sun [7, Theorem 4.1]. Throughout this section we fix $p \in \mathbb{N}$.

\section{AI Dynamics (I)}

Proof Recall the representation of the $p$-th moment of $u(0, t)$ in $(2.3)$, and the notation $\bar{X}^{\kappa}=\left(X_{1}^{\kappa}, X_{2}^{\kappa}, \ldots, X_{p}^{\kappa}\right)$. For $0 \leq s<t<\infty$ and $y, z \in \mathbb{R}^{d p}$ such that $y s, z t \in \mathbb{Z}^{d p}$, write

$$
\Xi_{y, z}^{\mathrm{STWN}}(s, t)=E_{y s}^{\otimes p}\left(\exp \left\{\sum_{1 \leqslant i<j \leqslant p} \int_{0}^{t-s} \mathbb{1}\left\{X_{i}^{\kappa}(v)=X_{j}^{\kappa}(v)\right\} d v\right\} \mathbb{1}\left\{\bar{X}^{\kappa}(t-s)=z t\right\}\right),
$$

where under $E_{y s}^{\otimes p}$ the process $\bar{X}^{\kappa}$ starts in $y s$. Abbreviate (in case it is well defined) $\Xi_{y}^{\mathrm{STWN}}(s, t)=\Xi_{y, y}^{\mathrm{STWN}}(s, t)$. It is enough to show the existence of a concave and symmetric function $\alpha: \mathbb{R}^{d p} \rightarrow \mathbb{R}$ such that, for all compact $K \subset \mathbb{R}^{d p}$,

$$
\lim _{t \rightarrow \infty} \sup _{y \in K t \cap \mathbb{Z}^{d p}}\left|\frac{1}{t} \log \Xi_{y / t}^{\mathrm{STWN}}(0, t)-\alpha(y / t)\right|=0 .
$$

Indeed, suppose that such a function exists. A short computation shows that $\alpha$ obtains a global maximum at zero. Moreover, a standard large deviation estimate for the number of jumps of $\bar{X}^{\kappa}$ shows that there is a compact subset $K \subset \mathbb{R}^{d p}$ such that

$$
\limsup _{t \rightarrow \infty} \frac{1}{t} \log E_{0}^{\otimes p}\left(\exp \left\{\sum_{1 \leq i<j \leq p} \int_{0}^{t} \mathbb{1}\left\{X_{i}^{\kappa}(v)=X_{j}^{\kappa}(v)\right\} d v\right\} \mathbb{1}\left\{\bar{X}^{\kappa}([0, t]) \subsetneq K t\right\}\right) \leq-1
$$

Hence, given such a set $K$, it is enough to focus on the contribution coming from those random walk paths such that $\left\{\bar{X}^{\kappa}[0, t] \subseteq K t\right\}$. Note that necessarily $0 \in K$. Fix $\varepsilon>0$. By the approximation property of $\alpha$ in (A.2) we can find a $t_{0} \geq 0$ such that, for all $t \geq t_{0}$,

$$
\alpha(0)-\varepsilon \leqslant \frac{1}{t} \log \sum_{\substack{y \in K t \\ y t \in \mathbb{Z}^{d p}}} \Xi_{y / t}^{\mathrm{sTwN}}(0, t) \leqslant \frac{1}{t} \log |K t|+\alpha(0)+\varepsilon,
$$

which yields the desired claim.

The proof of the existence of $\alpha$ is divided into 3 Steps. 
Step 1 We first show the existence of a function $\alpha: \mathbb{Q}^{d p} \rightarrow \mathbb{R}$ such that, for all $y \in \mathbb{Q}^{d p}$,

$$
\lim _{\substack{t \rightarrow \infty \\ y \in \mathbb{Z}^{d p}}} \frac{1}{t} \log \Xi_{y}^{\mathrm{STwN}}(0, t)=\alpha(y) .
$$

To that end, we fix $y \in \mathbb{Q}^{d p}$ and take $0 \leq s<u<t$ such that $y s, y u, y t \in \mathbb{Z}^{d p}$. Forcing $\bar{X}^{\kappa}$ to be at position $y u$ at time $u-s$, an application of the Markov property of $\bar{X}^{\kappa}$ at time $u-s$ yields

$$
\Xi_{y}^{\mathrm{STWN}}(s, t) \geqslant \Xi_{y}^{\mathrm{STWN}}(s, u) \Xi_{y}^{\mathrm{STWN}}(u, t) .
$$

Consequently, $t \mapsto \log \Xi_{y}^{\text {STwN }}(s, t)$ is superadditive for each $s$ as above, and the claim in (A.5) follows.

Step 2 To extend $\alpha$ to a function on $\mathbb{R}^{d p}$ and to get uniform convergence on compacts as in (A.2), we show that for any compact subset $K \subset \mathbb{R}^{d p}$,

$$
\lim _{\varepsilon \downarrow 0} \limsup _{t \rightarrow \infty} \sup _{\substack{x, y \in K, x t, y t \in \mathbb{Z}^{d p} \\\|x-y\| \leqslant \varepsilon}} \frac{1}{t}\left|\log \Xi_{x}^{\mathrm{STWN}}(0, t)-\log \Xi_{y}^{\mathrm{STWN}}(0, t)\right|=0 .
$$

To that end, we fix $\varepsilon>0$ and note that for all $t>0$ and all $y \in K$ such that $y t \in \mathbb{Z}^{d p}$,

$$
\Xi_{y}^{\mathrm{STWN}}(0, t)=\sum_{\substack{w \in \mathbb{R}^{d p} \\ w(1-\varepsilon) t \in \mathbb{Z}^{d p}}} \Xi_{w}^{\mathrm{STWN}}(0,(1-\varepsilon) t) \Xi_{w, y}^{\mathrm{STWN}}((1-\varepsilon) t, t) .
$$

Moreover, by standard large deviation estimates for the number of jumps for each component of $\bar{X}^{\kappa}$, it is possible to find an $R>0$ such that

$$
\limsup _{t \rightarrow \infty} \frac{1}{t} \log \sup _{y \in K} \sum_{\substack{w \notin B_{R} t \\ w(1-\varepsilon) t \in \mathbb{Z}^{d p}}} \Xi_{w}^{\mathrm{STwN}}(0,(1-\varepsilon) t) \Xi_{w, y}^{\mathrm{STwN}}((1-\varepsilon) t, t) \leqslant-1,
$$

so that the main contribution to (A.8) comes from those $w$ such that $w \in B_{R}$. Here, $B_{R}$ denotes the box centered at the origin with radius $R$. Consequently, to conclude Step 2 it is enough to show that

$$
\lim _{\varepsilon \downarrow 0} \limsup _{t \rightarrow \infty} \sup _{\substack{x, y \in K, x t, y t \in \mathbb{Z}^{d p} \\\|x-y\| \leqslant \varepsilon}} \frac{1}{t}\left|\log \frac{\sum_{w \in B_{R} t: w(1-\varepsilon) t \in \mathbb{Z}^{d p}} \Xi_{w}^{\mathrm{STWN}}(0,(1-\varepsilon) t) \Xi_{w, x}^{\mathrm{STWN}}((1-\varepsilon) t, t)}{\sum_{w \in B_{R} t: w(1-\varepsilon) t \in \mathbb{Z}^{d p}} \Xi_{w}^{\mathrm{STWN}}(0,(1-\varepsilon) t) \Xi_{w, y}^{\mathrm{STWN}}((1-\varepsilon) t, t)}\right|=0 .
$$

But this follows from the fact that the term appearing under the integral in the exponential in (2.3) is bounded, together with standard estimates on the random walk transition kernel. The details can be found in the proof of [7, Lemma 4.3].

Step 3 Using the results in Steps 1-2, we can conclude the proof as in [7]. We only give a sketch. Because of (A.7), $\alpha$ is continuous and hence can be extended to a 
continuous function $\alpha: \mathbb{R}^{d p} \rightarrow \mathbb{R}$. The uniform convergence in (A.2) follows from (A.7) and a compactness argument. Clearly, $\alpha$ is symmetric, i.e., $\alpha(x)=\alpha(-x)$ for all $x \in \mathbb{R}^{d p}$, which is a consequence of the symmetry of $\xi$. It remains to show the concavity of $\alpha$. For that, fix $x, y \in \mathbb{R}^{d p}, \beta \in(0,1)$ and take sequences $\left(t_{n}\right)_{n \in \mathbb{N}}$, $\left(x_{n}\right)_{n \in \mathbb{N}},\left(y_{n}\right)_{n \in \mathbb{N}}$ such that $\lim _{n \rightarrow \infty} t_{n}=\infty, \lim _{n \rightarrow \infty} x_{n} t_{n}=x, \lim _{n \rightarrow \infty} y_{n} t_{n}=y$, and $\beta y_{n} t_{n},(1-\beta) y_{n} t_{n} \in \mathbb{Z}^{d p}$ for all $n \in \mathbb{N}$. Then, constraining $\bar{X}^{\kappa}$ to be at position $\beta t_{n} y_{n}$ at time $\beta t_{n}$, we see that

$$
\log \Xi_{\beta y_{n}+(1-\beta) x_{n}}^{\mathrm{STWN}}\left(0, t_{n}\right) \geqslant \log \Xi_{y_{n}}^{\mathrm{STWN}}\left(0, \beta t_{n}\right)+\log \Xi_{y_{n}, \beta y_{n}+(1-\beta) x_{n}}^{\mathrm{STWN}}\left(\beta t_{n}, t_{n}\right) .
$$

The term in the left-hand side converges to $\alpha(\beta y+(1-\beta) x)$ after division by $t_{n}$, the first term in the right-hand side converges to $\alpha(y)$ after division by $\beta t_{n}$, while the second term in the right-hand side converges to $\alpha(x)$ after division by $(1-\beta) t_{n}$. This yields the existence of a function $\alpha$ as claimed in (A.2), and finishes the proof.

\section{A.2 Dynamics (IIa)}

Proof For $0 \leq s<t<\infty$ and $y, z \in \mathbb{R}^{d(n+p)}$ such that $y s, z t \in \mathbb{Z}^{d(n+p)}$, define

$$
\Xi_{y, z}^{\mathrm{FIRW}}(s, t)=\left(E^{\otimes n} \otimes E^{\otimes p}\right)_{y s}\left(\exp \left\{\sum_{i=1}^{p} \sum_{j=1}^{n} \int_{0}^{t-s} \mathbb{1}\left\{X_{i}^{\kappa}(v)=X_{j}^{\rho}(v)\right\} d v\right\} \mathbb{1}\{\bar{X}(t-s)=z t\}\right),
$$

where $\bar{X}=\left(X_{1}^{\kappa}, \ldots, X_{p}^{\kappa}, X_{1}^{\rho}, \ldots, X_{n}^{\rho}\right)$. The function $\alpha$ from (A.2) is constructed on $\mathbb{R}^{d(n+p)}$ rather than on $\mathbb{R}^{d p}$. The construction is similar to that for dynamics (I) and will therefore be omitted.

\section{A.3 Dynamics (IIb)}

Recall that the dynamics starts from a Poisson random field on $\mathbb{Z}^{d}$ with intensity $v \in$ $(0, \infty)$ and the representation derived in Section 2.4.2. For the proof we distinguish between two cases.

Case $p \geq 1 / G(0)$.

Proof For this case it is known that $\lambda_{p}^{\mathbb{1}}(\kappa)=\infty$ for all choices of $\kappa$, and hence it is enough to show that $\lambda_{0}^{\delta_{0}}(\kappa)=\infty$. However, this is a simple consequence of [9, Proposition 2.3 and Eq. (3.3)]. 
Case $0<p<1 / G(0)$.

Proof The proof works along similar lines as for (I). We only highlight the differences. For $0 \leq s<t<\infty$ and $y, t \in \mathbb{R}^{d p}$ such that $y s, z t \in \mathbb{Z}^{d p}$, define

$$
\Xi_{y, z}^{\mathrm{IIRW}}(s, t)=E_{y s}^{\otimes p}\left(\exp \left\{v \sum_{i=1}^{p} \int_{0}^{t-s} w\left(X_{i}^{\kappa}(v), v\right) d v\right\} \mathbb{1}\left\{\bar{X}^{\kappa}(t-s)=z t\right\}\right),
$$

where $\bar{X}^{\kappa}=\left(X_{1}^{\kappa}, \ldots, X_{p}^{\kappa}\right)$ and the process $\bar{X}^{\kappa}$ starts at $y s$ under $E_{y s}^{\otimes p}$. Abbreviate $\Xi_{y}^{\text {IRW }}(s, t)=\Xi_{y, y}^{\text {IIRW }}(s, t)$. It is again enough to establish a convergence similar to the one in (A.2), i.e., to show that there is a concave and symmetric function $\alpha: \mathbb{R}^{d p} \rightarrow$ $\mathbb{R}$ such that, for all compact subsets $K \subset \mathbb{R}^{d p}$,

$$
\lim _{t \rightarrow \infty} \sup _{y \in K t \cap \mathbb{Z}^{d p}}\left|\frac{1}{t} \log \Xi_{y / t}^{\mathrm{IRW}}(0, t)-\alpha(y / t)\right|=0 .
$$

The proof comes in 3 Steps and is similar to the proof for (I).

Step 1 Define the function $\alpha$ on $\mathbb{Q}^{d p}$ with the help of a superadditivity argument. To exhibit the dependence of the function $w$ on the trajectories $X_{1}^{\kappa}, \ldots, X_{p}^{\kappa}$ we write

$$
w(x, s)=w_{X_{1}^{\kappa}[0, t], \ldots, X_{p}^{\kappa}[0, t]}(x, s), \quad s \in[0, t] .
$$

It was argued in $[9,(4.11)]$ that, for all $s, t \geq 0$,

$w_{X_{1}^{\kappa}[0, s+t], \ldots, X_{p}^{\kappa}[0, t+s]}(x, u) \begin{cases}=w_{X_{1}^{\kappa}[0, s], \ldots, X_{p}^{\kappa}[0, s]}(x, u), & \text { for } u \in[0, s], \\ \geqslant w_{X_{1}^{\kappa}[s, s+t], \ldots, X_{p}^{\kappa}[s, s+t]}(x, u-s), & \text { for } u \in[s, s+t] .\end{cases}$

Therefore the superadditivity of $t \mapsto \log \Xi_{y}^{\mathrm{IRW}}(s, t)$ for each fixed value of $s$ follows in a similar fashion as for (I). This yields the existence of $\alpha$ on $\mathbb{Q}^{d p}$.

Step 2 As for (I), we want to show that, for any compact subset $K \subset \mathbb{R}^{d p}$,

$$
\lim _{\varepsilon \downarrow 0} \limsup _{t \rightarrow \infty} \sup _{\substack{x, y \in K, x t, y t \in \mathbb{Z}^{d} \\\|x-y\| \leqslant \varepsilon}} \frac{1}{t}\left|\log \Xi_{x}^{\text {IRW }}(0, t)-\log \Xi_{y}^{\text {IRW }}(0, t)\right|=0 .
$$

The difference with (I) is that we no longer have the same relation as in (A.8). However, by the lines following (2.29), we have the bound $w(x, t) \leq \bar{w}(0, t)$ for all $x \in \mathbb{Z}^{d}, t \geq 0$. Moreover, by (2.30), the assumption $0<p<1 / G(0)$ yields that $\bar{w}(0, t)$ is bounded. Hence, we can use large deviation arguments for the random walk to show that the main contribution to (A.13) comes from those random walk 
paths that stay until time $t$ inside a box of size $R t$ for a suitable chosen value of $R$. Moreover, using that, for all $t \geq 0, \varepsilon \in(0,1)$ and $x, y \in \mathbb{Z}^{d p}$,

$$
\begin{aligned}
& E_{0}^{\otimes p}\left(\mathbb{1}\left\{\bar{X}^{\kappa}((1-\varepsilon) t)=y\right\} \exp \left\{\sum_{i=1}^{p} \int_{0}^{(1-\varepsilon) t} w\left(X_{i}^{\kappa}(v), v\right) d v\right\}\right) P_{y}^{\otimes p}\left(\bar{X}^{\kappa}(t)=x\right) \\
& =E_{0}^{\otimes p}\left(\mathbb{1}\left\{\bar{X}^{\kappa}((1-\varepsilon) t)=y\right\} \exp \left\{\sum_{i=1}^{p} \int_{0}^{(1-\varepsilon) t} w\left(X_{i}^{\kappa}(v), v\right) d v\right\} \mathbb{1}\left\{\bar{X}^{\kappa}(t)=x\right\}\right) \\
& \leqslant E_{0}^{\otimes p}\left(\mathbb{1}\left\{\bar{X}^{\kappa}((1-\varepsilon) t)=y\right\} \exp \left\{\sum_{i=1}^{p} \int_{0}^{t} w\left(X_{i}^{\kappa}(v), v\right) d v\right\} \mathbb{1}\left\{\bar{X}^{\kappa}(t)=x\right\}\right) \\
& \leqslant \mathrm{e}^{p^{2} t G(0) /(1-p G(0))} E_{0}^{\otimes p}\left(\mathbb{1}\left\{\bar{X}^{\kappa}((1-\varepsilon) t)=y\right\} \exp \left\{\sum_{i=1}^{p} \int_{0}^{(1-\varepsilon) t} w\left(X_{i}^{\kappa}(v), v\right) d v\right\}\right) \\
& \quad \times P_{y}^{\otimes p}\left(\bar{X}^{\kappa}(t)=x\right),
\end{aligned}
$$

where we used (2.30) to obtain the last inequality and the relation (A.16) was used throughout all inequalities in (A.18). We can now proceed as for (I).

Step 3 This works almost verbatim as for (I). We omit the details.

\section{A.4 Dynamics (III)}

Proof The idea of the proof is the same as for (I)-(II), but some additional technical difficulties arise. Write $\mathbb{E}_{\mu, x}^{\otimes p}=\mathbb{E}_{\mu} \otimes E_{x}^{\otimes p}$ for the expectation when $\left(\xi, \bar{X}^{\kappa}\right)$, with $\bar{X}^{\kappa}=\left(X_{1}^{\kappa}, \ldots, X_{p}^{\kappa}\right)$ a collection of $p$ indendent simple random walks jumping at rate $2 d \kappa$, has initial distribution $\left(\mu, \delta_{x}\right)$. For $0 \leq s<t<\infty$ and $y, z \in \mathbb{R}^{d p}$ such that $y s, z t \in \mathbb{Z}^{d p}$, define, similarly as in (A.1),

$$
\Xi_{y, z}^{\mathrm{SFS}}(s, t)=\mathbb{E}_{\mu, y s}^{\otimes}\left(\exp \left\{\sum_{i=1}^{p} \int_{0}^{t-s} \xi\left(X_{i}^{\kappa}(v), v\right) d v\right\} \mathbb{1}\left\{\bar{X}^{\kappa}(t-s)=z t\right\}\right),
$$

and write $\Xi_{y}^{\mathrm{SFS}}(s, t)=\Xi_{y, y}^{\mathrm{SFS}}(s, t)$. As for $(\mathbf{I})$, it is enough to show the existence of a function $\alpha: \mathbb{R}^{d p} \rightarrow \mathbb{R}$ such that, for all compact subsets $K \subset \mathbb{R}^{d p}$,

$$
\lim _{t \rightarrow \infty} \sup _{y \in K t \cap \mathbb{Z}^{d}}\left|\frac{1}{t} \log \Xi_{y / t}^{\mathrm{SFS}}(0, t)-\alpha(y / t)\right|=0 .
$$

The proof comes in 3 Steps.

Step 1 We first show the existence of a function $\alpha: \mathbb{Q}^{d p} \rightarrow \mathbb{R}$ such that

$$
\lim _{\substack{t \rightarrow \infty \\ y t \in \mathbb{Z}^{d p}}} \frac{1}{t} \log \Xi_{y}^{\mathrm{SFS}}(0, t)=\alpha(y) .
$$


The idea is again to establish the superadditivity of $t \mapsto \log \Xi_{y}^{\mathrm{SFS}}(s, t)$ for all $y \in \mathbb{Q}^{d p}$ such that $y s, y t \in \mathbb{Z}^{d p}$. In the present context, however, this is a bit more tricky than before, which is why we provide the details. Fix $y \in \mathbb{Q}^{d p}$, and take $0 \leq s<u<t<$ $\infty$ such that $y s, y u, y t \in \mathbb{Z}^{d p}$. Constraining the random walk $\bar{X}^{\kappa}$ to be at position $y u$ at time $u-s$, we can use the strong Markov property of $\left(\xi, \bar{X}^{\kappa}\right)$ at time $u-s$ to get

$$
\Xi_{y}^{\mathrm{SFS}}(s, t) \geqslant \mathbb{E}_{\mu, y s}^{\otimes p}\left(\mathcal{E}(y u, u-s) \mathbb{E}_{\xi_{u-s}, y u}^{\otimes p}(\mathcal{E}(y t, t-u))\right),
$$

where we abbreviate

$$
\mathcal{E}(y, t)=\exp \left\{\sum_{i=1}^{p} \int_{0}^{t} \xi\left(X_{i}^{\kappa}(v), v\right) d v\right\} \mathbb{1}\left\{\bar{X}^{\kappa}(t)=y\right\}, \quad t \geqslant 0, y \in \mathbb{Z}^{d p} .
$$

Expanding the exponentials, we may rewrite the right-hand side of (A.22) as

$$
\begin{aligned}
\sum_{n, m \in \mathbb{N}_{0}}^{\infty} \frac{1}{n !} \frac{1}{m !}\left(\prod_{j=1}^{n}\right. & \left.\int_{0}^{u-s} d s_{j}^{(1)}\right)\left(\prod_{k=1}^{m} \int_{0}^{t-u} d s_{k}^{(2)}\right) \\
& \times \mathbb{E}_{\mu, y s}^{\otimes p}\left(\mathcal{H}\left(y u, s_{1}^{(1)}, \ldots, s_{n}^{(n)} ; u-s\right) \mathbb{E}_{\xi_{u-s}, y u}^{\otimes p}\left(\mathcal{H}\left(y t, s_{1}^{(2)}, \ldots, s_{m}^{(2)} ; t-u\right)\right)\right),
\end{aligned}
$$

where

$\mathcal{H}\left(y, s_{1}, \ldots, s_{n} ; t\right)=\prod_{j=1}^{n}\left[\sum_{i=1}^{p} \xi\left(X_{i}^{\kappa}\left(s_{j}\right), s_{j}\right)\right] \mathbb{1}\left\{\bar{X}^{\kappa}(t)=y\right\}, n \in \mathbb{N}, t, s_{1}, \ldots, s_{n} \geqslant 0, y \in \mathbb{Z}^{d p}$.

Note that by the non-negativity of $\xi$, for all $n \in \mathbb{N}, y u \in \mathbb{Z}^{d p}, s_{1}, \ldots, s_{n}, u-s \geq 0$,

$$
\mathcal{H}\left(y u, s_{1}, \ldots, s_{n} ; u-s\right)
$$

is a non-decreasing function of the $n p$-tuple $\left(\xi\left(X_{i}^{\kappa}\left(s_{j}\right), s_{j}\right), 1 \leq i \leq p, 1 \leq j \leq n\right)$. Hence, the attractiveness of $\xi$ implies that for all $m \in \mathbb{N}, y t \in \mathbb{Z}^{d p}, s_{1}, \ldots, s_{m}$, $t-u \geq 0$,

$$
\mathbb{E}_{\xi_{u-s}, y u}^{\otimes p}\left(\mathcal{H}\left(y t, s_{1}, \ldots, s_{m} ; t-u\right)\right)
$$

is a non-decreasing function of $\xi_{u-s}$. Therefore, since $\xi$ is positively correlated (recall (1.15)), Liggett [15, Corollary 2.21, Section II.2] yields that

$$
\begin{aligned}
\mathbb{E}_{\mu, y s}^{\otimes p} & \left(\mathcal{H}\left(y u, s_{1}^{(1)}, \ldots, s_{n}^{(n)} ; u-s\right) \mathbb{E}_{\xi_{u-s}, y u}^{\otimes p}\left(\mathcal{H}\left(y t, s_{1}^{(2)}, \ldots, s_{m}^{(2)} ; t-u\right)\right)\right) \\
& =E_{y s}^{\otimes p}\left[\mathbb{E}_{\mu}\left(\mathcal{H}\left(y u, s_{1}^{(1)}, \ldots, s_{n}^{(1)} ; u-s\right) \mathbb{E}_{\xi_{u-s}, y u}^{\otimes p}\left(\mathcal{H}\left(y t, s_{1}^{(2)}, \ldots, s_{m}^{(2)} ; t-u\right)\right)\right)\right] \\
& \geqslant E_{y u}^{\otimes p}\left[\mathbb{E}_{\mu}\left(\mathcal{H}\left(y u, s_{1}^{(1)}, \ldots, s_{n}^{(1)} ; u-s\right)\right) \mathbb{E}_{\mu \xi_{u-s}, y u}^{\otimes p}\left(\mathcal{H}\left(y t, s_{1}^{(2)}, \ldots, s_{m}^{(2)} ; t-u\right)\right)\right],
\end{aligned}
$$


where $\mu \xi_{u-s}$ is the distribution of $\xi$ at time $u-s$ when $\xi$ starts from $\mu$. But $\mu$ is an invariant measure, and so this distribution equals $\mu$. Consequently, the right-hand side of (A.28) becomes

$$
\mathbb{E}_{\mu, y s}^{\otimes p}\left(\mathcal{H}\left(y u, s_{1}^{(1)}, \ldots, s_{n}^{(1)} ; u-s\right)\right) \mathbb{E}_{\mu, y u}^{\otimes p}\left(\mathcal{H}\left(y t, s_{1}^{(2)}, \ldots, s_{m}^{(2)} ; t-u\right)\right) .
$$

Substituting (A.29) back into (A.24), we see that

$$
\Xi_{y}^{\mathrm{SFS}}(s, t) \geqslant \Xi_{y}^{\mathrm{SFS}}(s, u) \Xi_{y}^{\mathrm{SFS}}(u, t),
$$

from which the existence of $\alpha$ follows.

Step 2 As in the proof for (I), we want to establish that, for any compact subset $K \subset \mathbb{R}^{d}$,

$$
\lim _{\varepsilon \downarrow 0} \limsup _{t \rightarrow \infty} \sup _{\substack{x, y \in K, x t, y t \in \mathbb{Z}^{d} \\|| x-y|| \leqslant \varepsilon}} \frac{1}{t}\left|\log \Xi_{x}^{\mathrm{SFS}}(0, t)-\log \Xi_{y}^{\mathrm{SFS}}(0, t)\right|=0 .
$$

The difference with (I) is that we no longer have the same relation as in (A.8). However, because of the boundedness of $\xi$, we can use a large deviation argument for the random walk to show that the main contribution to (A.19) comes from those random walk paths that stay until time $t$ inside a box of size $R t$ for a suitable chosen value of $R$. Moreover, using that, for all $t \geq 0, \varepsilon \in(0,1)$ and $w, x \in \mathbb{Z}^{d p}$,

$$
\begin{aligned}
& \mathbb{E}_{\mu, 0}^{\otimes p}\left(\mathbb{1}\left\{\bar{X}^{\kappa}((1-\varepsilon) t)=w\right\} \exp \left\{\sum_{i=1}^{p} \int_{0}^{(1-\varepsilon) t} \xi\left(X_{i}^{\kappa}(v), v\right) d v\right\}\right) P_{w}^{\otimes p}\left(\bar{X}^{\kappa}(t)=x\right) \\
& =\mathbb{E}_{\mu, 0}^{\otimes p}\left(\mathbb{1}\left\{\bar{X}^{\kappa}((1-\varepsilon) t)=w\right\} \exp \left\{\sum_{i=1}^{p} \int_{0}^{(1-\varepsilon) t} \xi\left(X_{i}^{\kappa}(v), v\right) d v\right\} \mathbb{1}\left\{\bar{X}^{\kappa}(t)=x\right\}\right) \\
& \leqslant \mathbb{E}_{\mu, 0}^{\otimes p}\left(\mathbb{1}\left\{\bar{X}^{\kappa}((1-\varepsilon) t)=w\right\} \exp \left\{\sum_{i=1}^{p} \int_{0}^{t} \xi\left(X_{i}^{\kappa}(v), v\right) d v\right\} \mathbb{1}\left\{\bar{X}^{\kappa}(t)=x\right\}\right) \\
& \leqslant \mathrm{e}^{p \varepsilon t} \mathbb{E}_{\mu, 0}^{\otimes p}\left(\mathbb{1}\left\{\bar{X}^{\kappa}((1-\varepsilon) t)=w\right\} \exp \left\{\sum_{i=1}^{p} \int_{0}^{(1-\varepsilon) t} \xi\left(X_{i}^{\kappa}(v), v\right) d v\right\}\right) P_{w}^{\otimes p}\left(\bar{X}^{\kappa}(t)=x\right),
\end{aligned}
$$

we can finish the proof as for (I).

Step 3 Use the techniques from Step 1 to proceed in a similar manner as in Step 3 for (I). We omit the details.

\section{B A Technical Lemma}

The following lemma was used in Section 2.4.2. 
Lemma A.1 Let $L$ be the generator of the dynamics in (IIb). For $N \in \mathbb{N}$, define $V_{N}: \mathbb{N}_{0}^{\mathbb{Z}^{d}} \times \mathbb{Z}^{d} \rightarrow \mathbb{R}$ by $V_{N}(\eta, x)=\eta(x) \wedge N$ (recall $(2.12)$ ), and let $P_{t}^{V_{N}}$ be the semigroup of $\mathcal{L}^{V_{N}}=L+\Delta^{\mathcal{K}}+V_{N}$. Then for every $t>0$ there is a $g \in$ $L^{1}\left(\mathbb{N}_{0}^{\mathbb{Z}^{d}} \times \mathbb{Z}^{d}, \mu \otimes m\right)$ such that, for all $\eta \in \mathbb{N}_{0}^{\mathbb{Z}^{d}}$ and $y \in \mathbb{Z}^{d}$,

$$
\left|\left(\mathcal{L}^{V_{N}} P_{t}^{V_{N}} \bar{f}\right)(\eta, y) \times\left(P_{t}^{V_{N}} \bar{f}\right)(\eta, y)\right| \leqslant g(\eta, y)
$$

locally uniformly in $t$. Here, for $R>0, \bar{f}(\eta, y)=\mathbb{1}\left\{y \in B_{R}\right\}$ and $B_{R}$ is the box centered around the origin with radius $R$.

Proof We may assume that $t \geq 1$, which we do for notational convenience. It is straightforward to show that the statement is true when $\mathcal{L}^{V_{N}}=L+\Delta^{\mathcal{K}}+V_{N}$ in (B.1) is replaced by $\Delta^{\mathcal{K}}+V_{N}$. Furthermore, since $B_{R}$ is a finite set, it is enough to show that

$$
\left|\left(L P_{t}^{V_{N}} \delta_{w}\right)(\eta, y) \times\left(P_{t}^{V_{N}} \delta_{v}\right)(\eta, y)\right| \leqslant g(\eta, y)
$$

for any $v, w \in \mathbb{Z}^{d}$. For notational convenience we assume that $v=w=0$. The general case follows in a similar manner. Note that by the definition of $L$ in (1.11) we see that, for all $t>0$ and $(\eta, y) \in \mathbb{N}^{d} \times \mathbb{Z}^{d}$,

$$
\begin{aligned}
& \left|\left(L P_{t}^{V_{N}} \delta_{0}\right)(\eta, y) \times\left(P_{t}^{V_{N}} \delta_{0}\right)(\eta, y)\right| \\
& \quad \leqslant \sum_{x \in \mathbb{Z}^{d}} \sum_{z:\|z\|=1} \eta(x)\left|\left(P_{t}^{V_{N}} \delta_{0}\right)\left(\eta^{x, x+z}, y\right)-\left(P_{t}^{V_{N}} \delta_{0}\right)(\eta, y)\right| \mathrm{e}^{N t} P_{y}\left(X_{t}^{\mathcal{K}}=0\right) .
\end{aligned}
$$

To estimate the difference $\left|\left(P_{t}^{V_{N}} \delta_{0}\right)\left(\eta^{x, x+z}, y\right)-\left(P_{t}^{V_{N}} \delta_{0}\right)(\eta, y)\right|$, we introduce the following coupling.

Let $x \in \mathbb{Z}^{d}$ such that $\eta(x) \geq 1$, and let $\xi$ be (IIb) started in $\eta$ and $\xi^{x, x+z}$ be (IIb) started in $\eta^{x, x+z}$. Note that both systems start with the same number of simple random walks. Let $Y_{x}$ be simple random walk with jump rate $2 d$ started from $x$. We can couple $\xi$ and $\xi^{x, x+z}$ such that, for all $w \in \mathbb{Z}^{d}$ and $t \geq 0$,

$$
\xi^{x, x+z}(w, s)= \begin{cases}\xi(w, s), & w \neq Y_{x}(s), Y_{x}(s)+z \\ \xi(w, s)-1, & w=Y_{x}(s) \\ \xi(w, s)+1, & w=Y_{x}(s)+z\end{cases}
$$

With this coupling at hand, we see that

$$
\begin{aligned}
& \left|\left(P_{t}^{V_{N}} \delta_{0}\right)\left(\eta^{x, x+z}, y\right)-\left(P_{t}^{V_{N}} \delta_{0}\right)(\eta, y)\right| \\
& \quad \leqslant 2 \mathrm{e}^{N t} P_{y, x}\left(\exists s \in[0, t]: X^{\mathcal{K}}(s) \in\left\{Y_{x}(s), Y_{x}(s)+z\right\}, X^{\mathcal{K}}(t)=0\right),
\end{aligned}
$$


where $P_{y, x}$ denotes the product measure of $\left(X^{\mathcal{K}}, Y_{x}\right)$ started from $(y, x)$. Combining (B.3) and (B.5), we see that

$$
\begin{aligned}
& \sup _{s \in[t-1, t+1]}\left|\left(L P_{s}^{V_{N}} \delta_{0}\right)(\eta, y)\left(P_{s}^{V_{N}} \delta_{0}\right)(\eta, y)\right| \\
& \leqslant 2 \mathrm{e}^{2 N(t+1)} \\
& \times \sum_{x \in \mathbb{Z}^{d}} \sum_{z:\|z\|=1} \eta(x) P_{y, x}(\exists s \in[0, t+1]: \\
& \left.\quad X^{\mathcal{K}}(s) \in\left\{Y_{x}(s), Y_{x}(s)+z\right\}, 0 \in X^{\mathcal{K}}([t-1, t+1])\right) \\
& \quad \times P_{y}\left(\exists s \in[t-1, t+1]: X^{\mathcal{K}}(s)=0\right) .
\end{aligned}
$$

We complete the proof by showing that the right-hand side of (B.6) is in $L^{1}\left(\mathbb{N}_{0}^{Z^{d}} \times\right.$ $\left.\mathbb{Z}^{d}, \mu \otimes m\right)$. To see why, note that integration of the right-hand side of (B.6) over $\eta \in \mathbb{N}_{0}^{\mathbb{Z}^{d}}$ yields the upper bound

$$
\begin{aligned}
2 \sum_{x \in \mathbb{Z}^{d}} \sum_{z:\|z\|=1} v P_{y, x}\left(\exists s \in[0, t+1]: X^{\mathcal{K}}(s) \in\left\{Y_{x}(s), Y_{x}(s)+z\right\}, 0 \in X^{\mathcal{K}}([t-1, t+1])\right) \\
\times P_{y}\left(\exists s \in[t-1, t+1]: X^{\mathcal{K}}(s)=0\right) .
\end{aligned}
$$

Summing the right-hand side of (B.7) over $y \in \mathbb{Z}^{d}$, we get

$$
\begin{aligned}
2 \mathrm{e}^{2 N(t+1)} v & \sum_{x, y \in \mathbb{Z}^{d}} P_{y, x}\left(\exists s \in[0, t+1]:\left\|X^{\mathcal{K}}(s)-Y_{x}(s)\right\|=1,0 \in X^{\mathcal{K}}([t-1, t+1])\right) \\
& \times P_{y}\left(\exists s \in[t-1, t+1]: X^{\mathcal{K}}(s)=0\right) \leqslant I+I I
\end{aligned}
$$

where

$$
\begin{aligned}
I & =2 \mathrm{e}^{2 N(t+1)} \vee \sum_{y \in \mathbb{Z}^{d}} P_{y}\left(\exists s \in[t-1, t+1]: X^{\mathcal{K}}(s)=0\right), \\
I I & =2 \mathrm{e}^{2 N(t+1)} \vee \sum_{x \neq y} P_{y, x}\left(\exists s \in[0, t+1]:\left\|X^{\mathcal{K}}(s)-Y_{x}(s)\right\|=1,0 \in X^{\mathcal{K}}([t-1, t+1])\right) .
\end{aligned}
$$


The first property in (1.5) combined with standard large deviations estimates shows that $I$ is finite. To see that $I I$ is finite, note that by the Cauchy-Schwarz inequality,

$$
\begin{gathered}
I I \leqslant 2 \mathrm{e}^{2 N(t+1)} \sum_{y \in \mathbb{Z}^{d}} \sum_{k \in \mathbb{N}} \sum_{x:\|x-y\|=k} \sqrt{P_{y, x}\left(\exists s \in[0, t+1]:\left\|X^{\mathcal{K}}(s)-Y_{x}(s)\right\|=1\right)} \\
\times \sqrt{P_{y}\left(0 \in X^{\mathcal{K}}([t-1, t+1])\right)} .
\end{gathered}
$$

To proceed, note that for any $y \in \mathbb{Z}^{d}$,

$$
\begin{aligned}
\sum_{k \in \mathbb{N}} \sum_{x:\|x-y\|=k} \sqrt{P_{y, x}\left(\exists s \in[0, t+1]:\left\|X^{\mathcal{K}}(s)-Y_{x}(s)\right\|=1\right)} \\
\leqslant \sum_{k \in \mathbb{N}} \sum_{x:\|x-y\|=k} \sqrt{P_{y, x}\left(N\left(X^{\mathcal{K}}-Y_{x}, t+1\right) \geqslant k-1\right)} .
\end{aligned}
$$

Here, $N\left(X^{\mathcal{K}}-Y_{x}, t+1\right)$ denotes the number of jumps of $X^{\mathcal{K}}-Y_{x}$. Thus, the first property in (1.5) combined with standard large deviation estimates shows that the sum in (B.11) is bounded uniformly in $y$. To conclude, use that

$$
y \mapsto \sqrt{P_{y}\left(0 \in X^{\mathcal{K}}([t-1, t+1])\right)}
$$

decays faster than exponential in $\|y\|$. This implies that $I I$ is finite, and shows that the right-hand side of (B.6) is in $L^{1}\left(\mathbb{N}_{0}^{\mathbb{Z}^{d}} \times \mathbb{Z}^{d}, \mu \otimes m\right)$.

\section{References}

1. Andjel, E.D.: Invariant measures for the zero-range process. Ann. Probab. 10, 525-547 (1982)

2. Biskup, M.: Recent progress on the random conductance model. Prob. Surv. 8, 294-373 (2011)

3. Carmona, R.A., Molchanov, S.A.: Parabolic Anderson Problem and Intermittency AMS Memoirs 518. American Mathematical Society, Providence RI (1994)

4. Castell, F., Gün, O., Maillard, G.: Parabolic Anderson Model with a Finite Number of Moving Catalysts, Probability in Complex Physical Systems. In: Deuschel, J.-D., Gentz, B., König, W., Van Renesse, M.-K., Scheutzow, M., Schmock, U. (eds.) honour of Erwin Bolthausen and Jürgen Gärtner, pp. 91-117. Springer Proceedings in Mathematics 11, Springer, Berlin (2012)

5. Demuth, M., Van Casteren, J.A.: Stochastic Spectral Theory for Selfadjoint Feller Operators, A functional Integration Approach. Probability and Its Applications, Birkhäuser, Basel (2000)

6. Drewitz, A.: Lyapunov exponents for the one-dimensional parabolic Anderson model with drift. Electron. J. Probab. 13, 2283-2336 (2008)

7. Drewitz, A., Gärtner, J., Ramirez, A.F., Sun, R.: Survival probability of a random walk among a Poisson system of moving traps, same as [4], 119-158

8. Erhard, D., Den Hollander, F., Maillard, G.: The parabolic Anderson model in a dynamic random environment: basic properties of the quenched Lyapunov exponent. Ann. Inst. Henri Poincaré Probab. Stat. 50, 1231-1275 (2014)

9. Gärtner, J., Den Hollander, F.: Intermittency in a catalytic random medium. Ann. Probab. 34, 22192287 (2006) 
10. Gärtner, J., Den Hollander, F., Maillard, G.: Intermittency on catalysts: symmetric exclusion. Electron. J. Probab. 12, 516-573 (2007)

11. Gärtner, J., Den Hollander, F., Maillard, G.: Quenched Lyapunov exponent for the parabolic Anderson model in a dynamic random environment, same as [4], 159-193

12. Kingman, J.F.C.: Subadditive ergodic theory. Ann. Probab. 1, 883-909 (1973)

13. Kipnis, C., Landim, C.: Scaling Limits of Interacting Particle Systems Grundlehren Der Mathematischen Wissenschaften 320. Springer, Berlin (1999)

14. König, W., Salvi, M., Wolff, T.: Large deviations for the local times of a random walk among random conductances. Electron. Commun. Probab. 17, 1-11 (2012)

15. Liggett, T.M.: Interacting Particle Systems Grundlehren Der Mathematischen Wissenschaften 276. Springer, New York (1985) 